\title{
GUIDELINES
}

\section{EADSG Guidelines: Insulin Therapy in Diabetes}

\author{
Bahendeka Silver (D) Kaushik Ramaiya - Swai Babu Andrew • \\ Otieno Fredrick · Sarita Bajaj · Sanjay Kalra • Bavuma M. Charlotte • \\ Karigire Claudine $\cdot$ Anthony Makhoba
}

Received: January 30, 2018 / Published online: March 5, 2018

(c) The Author(s) 2018. This article is an open access publication

\section{EXECUTIVE SUMMARY AND RECOMMENDATIONS}

- A diagnosis of diabetes or hyperglycemia should be confirmed prior to ordering, dispensing, or administering insulin (A).

- Insulin is the primary treatment in all patients with type 1 diabetes mellitus (T1DM) (A).

- Typically, patients with T1DM will require initiation with multiple daily injections at the time of diagnosis. This is usually short-

Enhanced content To view enhanced content for this article go to https://doi.org/10.6084/m9.figshare. 5887030

B. Silver $(\bowtie) \cdot$ A. Makhoba

MKPGMS-Uganda Martyrs University | St. Francis

Hospital, Nsambya, Kampala, Uganda

e-mail: silverbahendeka@gmail.com

K. Ramaiya

Shree Hindu Mandal Hospital, Chusi Street, Dar es

Salaam, Tanzania

S. B. Andrew

Muhimbili University College of Health Sciences,

United Nations Road, Dar es Salaam, Tanzania

O. Fredrick

Department of Clinical Medicine and Therapeutics

School of Medicine, College of Health Science,

University of Nairobi, Nairobi, Kenya acting insulin or rapid-acting insulin analogue given 0 to $15 \mathrm{~min}$ before meals together with one or more daily separate injections of intermediate or long-acting insulin. Two or three premixed insulin injections per day may be used (A).

- The target glycated hemoglobin A1c (HbA1c) for all children with T1DM, including preschool children, is recommended to be $<7.5 \%(<58 \mathrm{mmol} / \mathrm{mol})$. The target is chosen aiming at minimizing hyperglycemia, severe hypoglycemia, hypoglycemic unawareness, and reducing the likelihood of development of longterm complications (B).

- For patients prone to glycemic variability, glycemic control is best evaluated by a

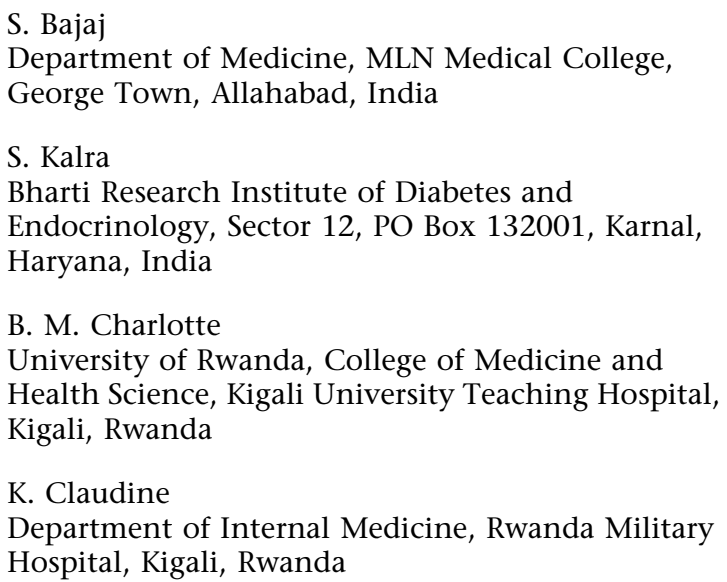


combination of results with self-monitoring of blood glucose (SMBG) (B).

- Indications for exogenous insulin therapy in patients with type 2 diabetes mellitus (T2DM) include acute illness or surgery, pregnancy, glucose toxicity, contraindications to or failure to achieve goals with oral antidiabetic medications, and a need for flexible therapy (B).

- In T2DM patients, with regards to achieving glycemic goals, insulin is considered alone or in combination with oral agents when $\mathrm{HbA} 1 \mathrm{c}$ is $\geq 7.5 \%$ ( $\geq 58 \mathrm{mmol} / \mathrm{mol})$; and is essential for treatment in those with $\mathrm{HbA} 1 \mathrm{c} \geq 10 \% \quad(\geq 86 \mathrm{mmol} / \mathrm{mol})$, when diet, physical activity, and other antihyperglycemic agents have been optimally used (B).

- The preferred method of insulin initiation in T2DM is to begin by adding a longacting (basal) insulin or once-daily premixed/co-formulation insulin or twicedaily premixed insulin, alone or in combination with glucagon-like peptide-1 receptor agonist (GLP-1 RA) or in combination with other oral antidiabetic drugs (OADs) (B).

- If the desired glucose targets are not met, rapid-acting or short-acting (bolus or prandial) insulin can be added at mealtime to control the expected postprandial raise in glucose. An insulin regimen should be adopted and individualized but should, to the extent possible, closely resemble a natural physiologic state and avoid, to the extent possible, wide fluctuating glucose levels (C).

- Blood glucose monitoring is an integral part of effective insulin therapy and should not be omitted in the patient's care plan. Fasting plasma glucose (FPG) values should be used to titrate basal insulin, whereas both FPG and postprandial glucose (PPG) values should be used to titrate mealtime insulin (B).

- Metformin combined with insulin is associated with decreased weight gain, lower insulin dose, and less hypoglycemia when compared with insulin alone (C).
- Oral medications should not be abruptly discontinued when starting insulin therapy because of the risk of rebound hyperglycemia (D).

- Analogue insulin is as effective as human insulin but is associated with less postprandial hyperglycemia and delayed hypoglycemia (B).

- The shortest needles (currently the 4-mm pen and 6-mm syringe needles) are safe, effective, and less painful and should be the first-line choice in all patient categories; intramuscular (IM) injections should be avoided, especially with longacting insulins, because severe hypoglycemia may result; lipohypertrophy is a frequent complication of therapy that distorts insulin absorption, and therefore, injections and infusions should not be given into these lesions and correct site rotation will help prevent them (A).

- Many patients in East Africa reuse syringes for various reasons, including financial. This is not recommended by the manufacturer and there is an association between needle reuse and lipohypertrophy. However, patients who reuse needles should not be subjected to alarming claims of excessive morbidity from this practice (A).

- Health care authorities and planners should be alerted to the risks associated with syringe or pen needles $6 \mathrm{~mm}$ or longer in children (A).

Keywords: Diabetes mellitus; East Africa; Guidelines; Hyperglycemia; Hypoglycemia; Insulin therapy; Type 1 diabetes mellitus (T1DM); Type 2 diabetes mellitus (T2DM)

\section{INTRODUCTION}

Based on the most recent International Diabetes Federation (IDF) report, the number of people with diabetes will increase from 425 million in 2017 to 629 million by 2045 [1], with approximately $80 \%$ of the people affected by diabetes residing in low- and middle-income countries 
(LMIC). These countries are already burdened by infectious diseases and scarce human and financial resources [2], emphasizing the importance of contextually appropriate and timely treatment of diabetes in these communities.

The importance of glycemic control in preventing and delaying the progression of diabetes complications is well established [3-5]. Indeed, the last decade has experienced considerable efforts undertaken in introducing new classes of glucose-lowering medications and formulating guidelines for the use of these therapies to optimize glycemic control [6]. However, insulin therapy remains the most widely relied upon as the mainstay therapy for diabetes [7].

Current trends on glycemic control look at several composite glycemic end points rather than individual itemized goals of measured glucose levels. This has given rise to the concept of glycemic pentads and glycemic hexads [8].

\section{GLYCEMIC CONTROL IN DIABETES}

\section{Glycemic Hexads}

The terms glycemic pentads and glycemic hexads have been introduced to explain the importance of safely achieving tight glucose control [8]. The efficacy and safety objectives of the pharmacologic intervention in diabetes management need to consider the individual patient needs, fears, and comorbidity factors among others. The concept of glycemic hexads includes three efficacy parameters, namely glycosylated hemoglobin A1c (HbA1c), fasting plasma glucose (FPG), and postprandial plasma glucose (PPG), along with three safety parameters, namely hypoglycemia in general, nocturnal hypoglycemia (in special situations), and glycemic variability. Nocturnal hypoglycemia is reported as an episode of abnormally low blood glucose $(3.5 \mathrm{mmol} / \mathrm{L})$ occurring at nighttime during sleep, especially in patients with type 1 diabetes mellitus (T1DM). In the 4 years of follow-up after the Diabetes Control and Complications Trial (DCCT), 43\% of all hypoglycemic episodes and $55 \%$ of severe episodes were reported to occur during sleep [9]. Patients with type 2 diabetes mellitus (T2DM) treated with long-acting sulfonylureas (SUs), insulin, or a combination of both are also susceptible to nocturnal hypoglycemia. Glycemic variability is a surrogate that explains the association between hyperglycemia and increased cardiovascular $(\mathrm{CV})$ risk in persons affected by diabetes. Efforts should be made to minimize glycemic variability so as to prevent future $\mathrm{CV}$ events [8].

\section{Target Values for Glycemic Control}

The primary objective in the management of diabetes is to reduce high blood glucose levels sufficiently to relieve any symptoms of hyperglycemia and to prevent/delay the onset of diabetes complications. Several surrogate markers for this important outcome have been studied: FPG, 2-h PPG, fructosamine measurements, glycated albumin, and HbA1c [10]. The HbA1c is a good surrogate marker for the longterm glycemic control [10]. In T2DM with elevated blood glucose level, high HbA1c at the time of presentation predicts a significantly increased risk of microvascular and macrovascular diseases $[11,12]$.

Intensive glycemic control with $\mathrm{HbA1c}$ target level of $6.4-7.1 \% \quad(46-54 \mathrm{mmol} / \mathrm{mol})$ is associated with reduction in risk of microvascular disease as reported by landmark trials: United Kingdom Prospective Diabetes Study (UKPDS) [4], the Kumamoto study [13], Action in Diabetes and Vascular Disease: Preterax and Diamicron MR Controlled Evaluation (ADVANCE) [14], Action to Control Cardiovascular Risk in Diabetes (ACCORD) [15], and Veterans Affairs Diabetes Trial (VADT) [16]. However, the benefits of intensive therapy should be weighed against the increase in total and cardiovascular disease (CVD)-related mortality, increased weight gain, and high risk for severe hypoglycemia [15].

The recommended HbA1c target in most patients with diabetes is $<7 \%(<53 \mathrm{mmol} / \mathrm{mol})$; in newly diagnosed patients with diabetes it is $<6.5 \%(<48 \mathrm{mmol} / \mathrm{mol})$, and in patients with diabetes who are weak with multiple comorbidities, including CVD and advanced renal 
disease and high hypoglycemic unawareness, it is between $7.1 \%(54 \mathrm{mmol} / \mathrm{mol})$ and $8.5 \%$ ( $69 \mathrm{mmol} / \mathrm{mol}$ ). For palliative care, the aim is to avoid symptomatic hyperglycemia.

The American Association of Clinical Endocrinologists and American College of Endocrinology (AACE/ACE) recommend an FPG target of 5.5-6.9 mmol/L [17]. It has been found that in nondiabetic individuals, the peak PPG generally does not exceed $7.8 \mathrm{mmol} / \mathrm{L}$. However, a linear progression exists between the postload glucose level and CVD with no "lower" limit cutoff [18]. The IDF recommends a PPG target of $<9.0 \mathrm{mmol} / \mathrm{L}$ whereas the American Diabetes Association (ADA) recommends a PPG target of $<10 \mathrm{mmol} / \mathrm{L}$ [10]. Overall, the glycemic control is dependent on the HbA1c target. It is recommended in patients with stable glycemic control that HbA1c needs to be monitored at least every 6 months, while in patients who are not at target and in whom interventions have intensified, HbA1c needs monitoring at 3-month intervals.

\section{Recommendation for Target Values in Type 1 Diabetes Mellitus}

- The target glycated hemoglobin A1c (HbA1c) for all children with type 1 diabetes mellitus (T1DM), including preschool children, is recommended to be $<7.5 \%(<58 \mathrm{mmol} / \mathrm{mol})$ (Grade B, EL I)

\section{Long-Term Glycemic and Metabolic Control}

Early in the 1930s, in the first textbook on diabetes, The Treatment of Diabetes Mellitus, Dr. Elliot Proctor Joslin, MD advocated that diabetes should be diagnosed early and the condition should be treated vigorously through the use of carbohydrate-restricted diets and fasting and regular exercise [19]. Since then, there has been an enormous body of evidence showing that early management of diabetes with tight glucose control, i.e., keeping blood glucose levels as close to normal as possible, is vital in the prevention of short- and long-term complications of diabetes. In short, Dr. Elliot was advocating for the Hit Early and Hit Hard paradigm shift in the management of diabetes. In East African discourse, this would be hit early and hit hard, like hitting a snake at first sight. There are, however, still areas of controversy regarding which tools to use in achieving the tight glycemic control, and in how tight is tight enough $[9,14,18,20]$. The following section explores the evidence available in the various approaches and how they can be fully utilized in the East African context against the background that the prevalence of diabetes and hypertension has increased in spite of a relatively low prevalence of dyslipidemia and obesity in this population [21].

\section{Recommendations for Target Values in Nonpregnant Adults with Type 2 Diabetes Mellitus (T2DM)}

- Fasting plasma glucose (FPG): 4.4-$7.2 \mathrm{mmol} / \mathrm{L}$

- Postprandial plasma glucose (PPG): $<10.0 \mathrm{mmol} / \mathrm{L}$

- 2-h postprandial plasma glucose (2-h PPG): $<7.8 \mathrm{mmol} / \mathrm{L}$

- HbA1c:

- $<6.5 \%(<48 \mathrm{mmol} / \mathrm{mol})$ in newly diagnosed patients with T2DM; those treated with lifestyle or metformin only; T2DM with long life expectancy or no significant cardiovascular $(\mathrm{CV})$ disease.

- $<7.0 \%(<53 \mathrm{mmol} / \mathrm{mol})$ in most $\mathrm{DM}$ patients.

- $<8.0 \%(<64 \mathrm{mmol} / \mathrm{mol})$ in patients with history of severe hypoglycemia; limited life expectancy; advanced microvascular or macrovascular complications; or longstanding diabetes in whom the goal is difficult to achieve despite diabetes selfmanagement education, appropriate glucose monitoring, and effective doses of multiple antihyperglycemic therapy including insulin.

- For palliative care, the aim is to avoid symptomatic hyperglycemia (Grade A, EL I) 
- Hypoglycemia/nocturnal hypoglycemia/glycemic variability: Achieving glycemic targets while minimizing

glucose variability and hypoglycemia, particularly major and nocturnal hypoglycemia, is of much importance while considering insulin therapy (Grade A, EL I)

Studies from Tanzania indicate a high morbidity and mortality associated with diabetes in the region in 2017 [1]. This may be a consequence of the health system being overburdened by infectious diseases, including human immunodeficiency virus infection and acquired immune deficiency syndrome (HIV/AIDS), tuberculosis (TB), and malaria. There are significant challenges in accessing diagnosis and treatment for diabetes, further complicated by social, cultural, and ethnic factors.

In general, diabetes in East Africa is characterized by a significant number of affected individuals remaining undiagnosed, and when the diagnosis is made, it is with late presentation with the majority subsequently not accessing appropriate care [1, 22-30]. Hence, complications of diabetes are common and increasingly more patients require insulin therapy. In addition, there are challenges of accessing medicines including insulin and associated technologies [31].

Socio-politico-economic realities present challenges to individualized care in most parts of East Africa. These challenges include poverty of the population, low national spending on health, significant out-of-pocket health expenditures, medication stock-outs, and lack of facilities and equipment [32-34].

\section{Current Status of Diabetes Management in East Africa}

The first description of diabetes in East Africa is attributed to Sir A.R. Cook [35], who, in 1901, reported that diabetes was rare in Uganda, but when encountered, it was fatal. A century later, diabetes is no longer rare, but is still fatal.
Presentation of T2DM in East Africa is still characterized by acute complications superimposed on late chronic complications of diabetes, resulting mainly from delay in accessing health care [36]. Recent added challenges in the management of diabetes include comorbidity with HIV, tuberculosis, malaria, and depression. T1DM and other forms of diabetes have a similar landscape. T1DM has previously been reported as rare, probably because of being misdiagnosed. Current data on T1DM reveals an increasing incidence and prevalence, and unfortunately with high rates of complications and premature mortality [37-39].

\section{Inpatient Settings in East Africa}

Hospitalization for hyperglycemia is often associated with prolonged stay, rehospitalization, and increased mortality [29]. Challenges with in-hospital hyperglycemia (IHH) management include lack of trained staff, cultural practices (such as not taking Western medicine for abscesses), beliefs in witchcraft, and traditional medicines [40]. The majority would have diabetes just detected or would have failed on oral hypoglycemic agents or stopped medications. Insulin therapy is required for most of these patients.

\section{Ambulatory Patients (Outpatient Services) in East Africa}

In East Africa, ambulatory patients receive a less intensive treatment protocol for hyperglycemia with delayed insulin treatment because of multiple barriers that include availability of insulin and associated delivery devices; patients' fear regarding insulin therapy; and physicians' concerns for the management of primary pathology, lack of monitoring devices, poor knowledge in medical nutritional therapy, and fear of hypoglycemia [41].

\section{Critical Care in East Africa}

The majority of health care facilities in East Africa do not have intensive care units (ICU). Very ill patients with diabetes are managed in high dependency units or, as in most cases, general wards. The majority of this category of patients require insulin therapy. 


\section{Diabetes Complications in East Africa}

In East Africa, short- and long-term complications of diabetes are very frequently encountered [38, 39, 42-45]. This has been attributed to multiple causes, ranging from late presentation to unavailability of services and cultural practices [29]. A significant number of patients present with long-term complications at diagnosis including diabetic foot ulcers leading to amputation [46].

\section{INSULIN OVERVIEW}

\section{Normal Insulin Physiology}

In healthy individuals, plasma glucose concentrations keep within a narrow range of about 3.5-7.0 mmol/L throughout the day despite wide fluctuations in nutritional intake, physical exercise, and other physiological, psychological, and iatrogenic determinants of plasma glucose concentrations. After food intake, plasma glucose rises to a peak in 30-60 min and returns to basal or below basal concentrations within $2-3 \mathrm{~h}$. In healthy individuals, this is achieved by an appropriate response of insulin production from the $\beta$-cells of the pancreas. Approximately $50 \%$ of the total daily insulin is secreted during basal periods, suppressing lipolysis and glycogenolysis. The remainder of insulin secretion is postprandial. In response to a meal, there is a rapid and sizable release of preformed insulin from storage granules within the $\beta$-cell. This is referred to as the first phase of insulin secretion [47]. This first phase of insulin secretion promotes peripheral utilization of the prandial nutrient load, suppresses hepatic glucose production, and limits postprandial glucose elevation $[48,49]$. The first phase of insulin secretion begins within $2 \mathrm{~min}$ of nutrient

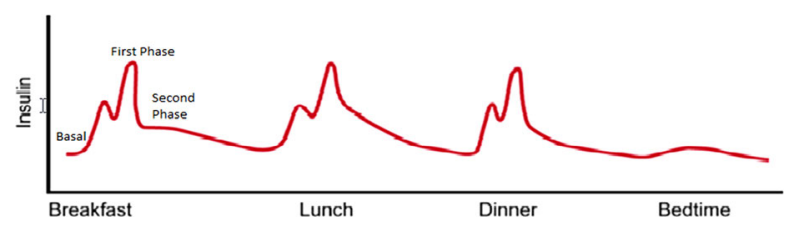

Fig. 1 Phases of normal insulin secretion Modified from [49] ingestion and continues for 10-15 min, giving way to the second phase of insulin secretion. The second phase of prandial insulin secretion is sustained until normoglycemia is restored. This is pictorially shown in Fig. 1. It is the loss of $\beta$-cells that underlies type 1 diabetes mellitus, and loss of $\beta$-cell glucose sensitivity and responsiveness that underlies the pathogenesis of T2DM. Between the $\beta$-cell loss and ineffective insulin release and function lie other forms of diabetes.

\section{Guidelines on Insulin Therapy}

\section{General Objectives}

To provide guidelines for clinical practice on the use of insulin in diabetes based on the best available evidence to health care workers; and for the rational use of resources in the diagnosis, treatment, and follow-up of diabetes in the East African population with diabetes.

\section{Specific Objectives}

To improve the rational use of insulin therapy in persons presenting with diabetes who require insulin treatment.

To indicate the appropriate pharmacological interventions in the context of multifactorial treatment plan, emphasizing cost-effectiveness, individualized goals, and safety.

Key areas in insulin therapy discussed in the guidelines include insulin regimen in patients with T1DM, a stepwise approach to insulin initiation, titration, and intensification in patients with T2DM, self-monitoring of blood glucose (SMBG), awareness and management of hypoglycemia, weight gain with intensive insulin therapy, and the role of psychosocial aspects associated with insulin therapy in diabetes care.

Areas beyond insulin therapy, but within the context of improving diabetes care, that have been included in the guidelines are lifestyle modifications and target values for glucose control based on the glycemic hexads (HbA1c, FPG, PPG, hypoglycemia, nocturnal hypoglycemia, and glycemic variability) [8]. The guidelines have gone further to include a strategic treatment approach to special 
populations such as diabetes in pregnancy; diabetes and lactation; diabetes and renal, cardiac, and hepatic impairment; and monogenic diabetes. A discussion on the management of diabetes in special situations like Ramadan and other faith-based fasting has been included. Furthermore, diabetes management during acute and chronic infections has been included since infections may be associated with adverse outcomes in diabetes management.

\section{METHODOLOGY AND EVIDENCE}

In drawing up the East African Diabetes Study Group (EADSG) Guidelines: Insulin Therapy in Diabetes, the authors adhered to the international and ethical standards for developing clinical practice guidelines (CPG) $[41,50]$. In compliance with the Uganda National Council of Science and Technology, the photo shown in Fig. 2 was taken with the written consent of the patient embedded in his clinical notes and is anonymized. This guideline is based on previously conducted studies and does not contain any studies with human participants or animals performed by any of the authors. A systematic review of existing guidelines and select literature from medical databases (MEDLINE) and African Journals
Online (AJOL) for relevant abstracts on insulin therapy was performed. The search used the medical subject headings (MeSH) terms "Insulin", "Rapid-Acting Insulin", "Short-Acting Insulin", "Intermediate-Acting Insulin", "Long-Acting Insulin", "Beef Insulin", "Pork Insulin", "Insulin analogues", "East Africa", "Burundi", "Kenya", "Rwanda", "Tanzania", and "Uganda" in English language without date restrictions. Abstracts of all the eligible papers in English were reviewed independently and articles were considered for inclusion if they met the following criteria: metaanalysis, systematic reviews, randomized controlled trials (RCT) [phase I-IV], case reports/series, and expert opinion in the management of diabetes. Exclusion criteria were (1) studies that are not published in English language and (2) studies that did not have full papers.

The recommendations for lifestyle modification, target values for glucose control, glycemic hexads targets, stepwise approach such as insulin initiation, titration, and intensification in diabetes patients, SMBG, hypoglycemia, weight gain, and psychosocial aspects, and recommendations in special populations and special situations in Eastern Africa regions were presented, debated, and appraised by the EADSG Guidelines Development Task Force at two meetings held in November 2017 and
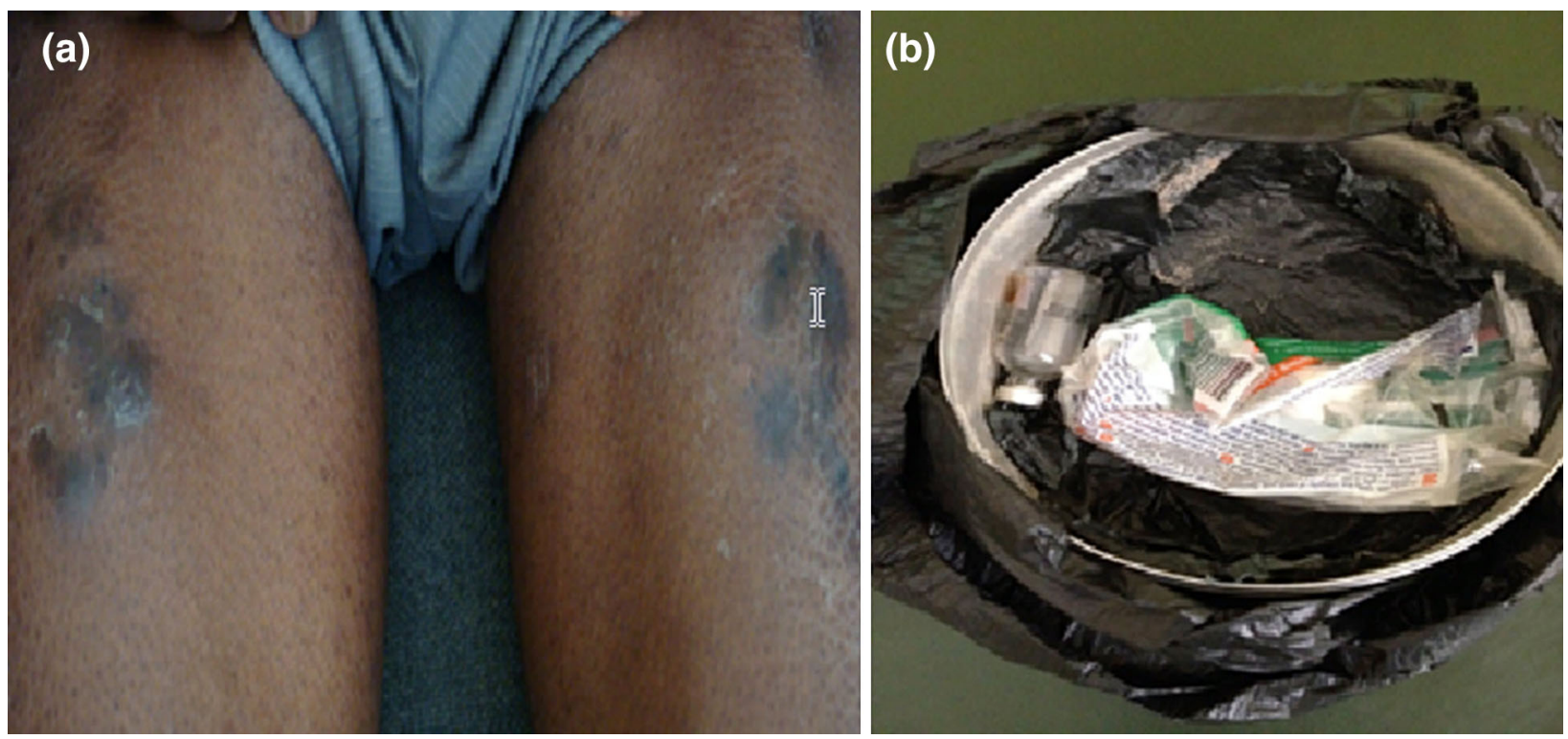

Fig. 2 Insulin injection tattoos (a) formed as a result of overslanting the needle and injecting contaminated insulin resulting from storing it in a water container (b) Image courtesy of Silver Bahendeka 
January 2018 and based on the current global guidelines [10, 51-55].

Indications for the initiation of insulin therapy and the type of insulin to be used (human vs analogues) along with advantages and disadvantages of each insulin regimen were extensively discussed. Where there was insufficient evidence, the EADSG Guidelines Development Task Force resorted to an evidencebased consensus to arrive at the guideline. The guidelines were drafted and circulated for further review by the group members and other external reviewers within and outside Africa. The EADSG Guidelines: Insulin Therapy in Diabetes document was finalized as a simple, unbiased, and relevant guideline for the Eastern African settings to ensure patient values as a base for all clinical decisions.

The hierarchical system of classifying the evidence is used in these guidelines [56] where applicable, including the levels of evidence

Table 1 Level of evidence

\begin{tabular}{|c|c|}
\hline Level & Type of evidence \\
\hline IA & Systematic review (with homogeneity) of RCTs \\
\hline IB & Individual RCT (with narrow $\mathrm{CI}$ ) \\
\hline IC & All or none RCT \\
\hline IIA & $\begin{array}{l}\text { Systematic review (with homogeneity) of cohort } \\
\text { studies }\end{array}$ \\
\hline IIB & $\begin{array}{l}\text { Individual cohort study (including low quality } \\
\text { RCT, e.g., }<80 \% \text { of follow-up) }\end{array}$ \\
\hline IIC & “Outcomes” research; ecological studies \\
\hline IIIA & $\begin{array}{l}\text { Systematic review (with homogeneity) of } \\
\text { case-control studies }\end{array}$ \\
\hline IIIB & Individual case-control study \\
\hline IV & $\begin{array}{l}\text { Case series (poor quality cohort and case-control } \\
\text { study) }\end{array}$ \\
\hline $\mathrm{V}$ & $\begin{array}{l}\text { Expert opinion without explicit critical appraisal or } \\
\text { based on physiological bench research or "first } \\
\text { principles" }\end{array}$ \\
\hline
\end{tabular}

$R C T$ randomized controlled trial, $C I$ confidence interval
(LoE) (Table 1) and grade practice recommendations (Table 2).

\section{Insulin Therapy}

Soon after the discovery of insulin in the early 1920s, both patients and health workers looked at this as a step towards the cure of diabetes, despite that insulin was a replacement therapy with no curative effects on the chronic state of diabetes [57]. This desire for a cure of diabetes is still a concern for many patients with diabetes; and for many of them, insulin therapy belongs to the category of just a Band-Aid solution. This, unfortunately, impacts negatively on insulin therapy. In spite of this, insulin therapy over the years has been revolutionized, leading to new improved formulations on the market, and devices to administer and monitor its effect [58]. Table 3 displays insulins available in East Africa. Some insulin analogues are available in pharmacies in East Africa, but are not yet on the purchase lists of governments; patients have therefore to pay out-of-pocket to access them. Moreover, their use worldwide is associated with increased cost of managing diabetes and they are thus further discouraged in low-income areas like East African countries [59]. Consequently there is no subsidy on these insulins and the full cost has to be borne by the patient. There is no published data on insurance with regards to insulin prescribing in East Africa but information obtained from dispensing pharmacies indicates that insurance companies do cover the costs when insulin analogues are prescribed.

\section{Recommendation}

- Insulin analogues are available in East Africa and may be safely prescribed where appropriate, taking into consideration the cost and the benefits gained when compared with human insulin that is cheaper and readily available (Grade $\mathbf{D}, \mathbf{E L}$ V)

Various terminologies relating to the use of insulin have been used: augmentation therapy refers to the addition of basal insulin to a 
Table 2 Grade practice recommendations

\begin{tabular}{|c|c|c|c|}
\hline Grade & Descriptor & Quantifying evidence & Implications for practice \\
\hline A & $\begin{array}{l}\text { Strong } \\
\quad \text { recommendation }\end{array}$ & $\begin{array}{l}\text { Level I evidence or consistent } \\
\text { findings from multiple studies of } \\
\text { levels II, III, or IV }\end{array}$ & $\begin{array}{l}\text { Clinicians should follow a strong recommendation } \\
\text { unless a clear and compelling rationale for an } \\
\text { alternative approach is present }\end{array}$ \\
\hline $\mathrm{B}$ & Recommendation & $\begin{array}{l}\text { Levels II, III, or IV evidence and } \\
\text { findings are generally consistent }\end{array}$ & $\begin{array}{l}\text { Generally, clinicians should follow a recommendation } \\
\text { but should remain alert to a new information and } \\
\text { sensitive to patient preferences }\end{array}$ \\
\hline $\mathrm{C}$ & Option & $\begin{array}{l}\text { Levels II, III, or IV evidence but } \\
\text { findings are inconsistent }\end{array}$ & $\begin{array}{l}\text { Clinicians should be flexible in their decision-making } \\
\text { regarding appropriate practice, although they may } \\
\text { set bounds on alternatives; patient preference } \\
\text { should have a substantial influencing role }\end{array}$ \\
\hline $\mathrm{D}$ & Option & $\begin{array}{l}\text { Level V evidence: little or no } \\
\text { systematic empirical evidence }\end{array}$ & $\begin{array}{l}\text { Clinicians should consider all options in their } \\
\text { decision-making and be alert to new published } \\
\text { evidence that clarifies the balance of benefit versus } \\
\text { harm; patient preference should have a substantial } \\
\text { influencing role }\end{array}$ \\
\hline
\end{tabular}

regimen when there is still some $\beta$-cell function present while replacement therapy refers to the use of a regimen that mimics the normal physiology of insulin secretion and is required when there is $\beta$-cell exhaustion. Rescue therapy refers to the use of replacement regimens for several weeks usually to reverse glucose toxicity [60]. Insulin regimens are difficult to classify, and for future comparisons of regimes of insulin in East Africa, we suggest to adopt the classification proposed by Neu et al. [61], which is based on three categories: (1) fixed insulin dose regimens, (2) glucose and meal-adjusted regimens, and (3) pump therapy. Insulins that may be used in the regimens include short-acting insulin, basal (long-acting) insulin, and premixed insulin. Blood glucose monitoring is an integral part of insulin therapy and it guides the regimens [62]. Measuring and recording both fasting and 1- or 2-h PPG levels over a 2- to 3 -day period is the first step in pattern management. The patient's insulin intake is determined by the pattern of these values, with adjustments made for anticipated need.

All patients on insulin therapy together with their caregivers require appropriate education on insulin therapy. Five basic principles of insulin management have been advised [62]: (1) insulin doses should not be skipped. Therefore to avoid high blood glucose levels caused by low or missed doses, short-acting insulin should be given every $6 \mathrm{~h}$, in four equal doses, or rapidacting insulin before each meal with a longacting basal insulin; (2) routine daily regimens should reflect the pattern of PPG levels over the previous 2 or 3 days; (3) rapid-acting insulin doses should be based primarily on the amount to be eaten, rather than on premeal glucose levels (although abnormally elevated or depressed levels may require correction); (4) parameters for glucose levels should be set and patients instructed to call (or to administer a correction dose) if the value falls above or below a predetermined range; and (5) to consider providing patients on insulin therapy (or their parents, school nurse, hospital staff, or other health care worker) with an algorithm that uses a basal insulin dose and premeal rapid-acting insulin doses, adjusted for caloric or carbohydrate intake. 


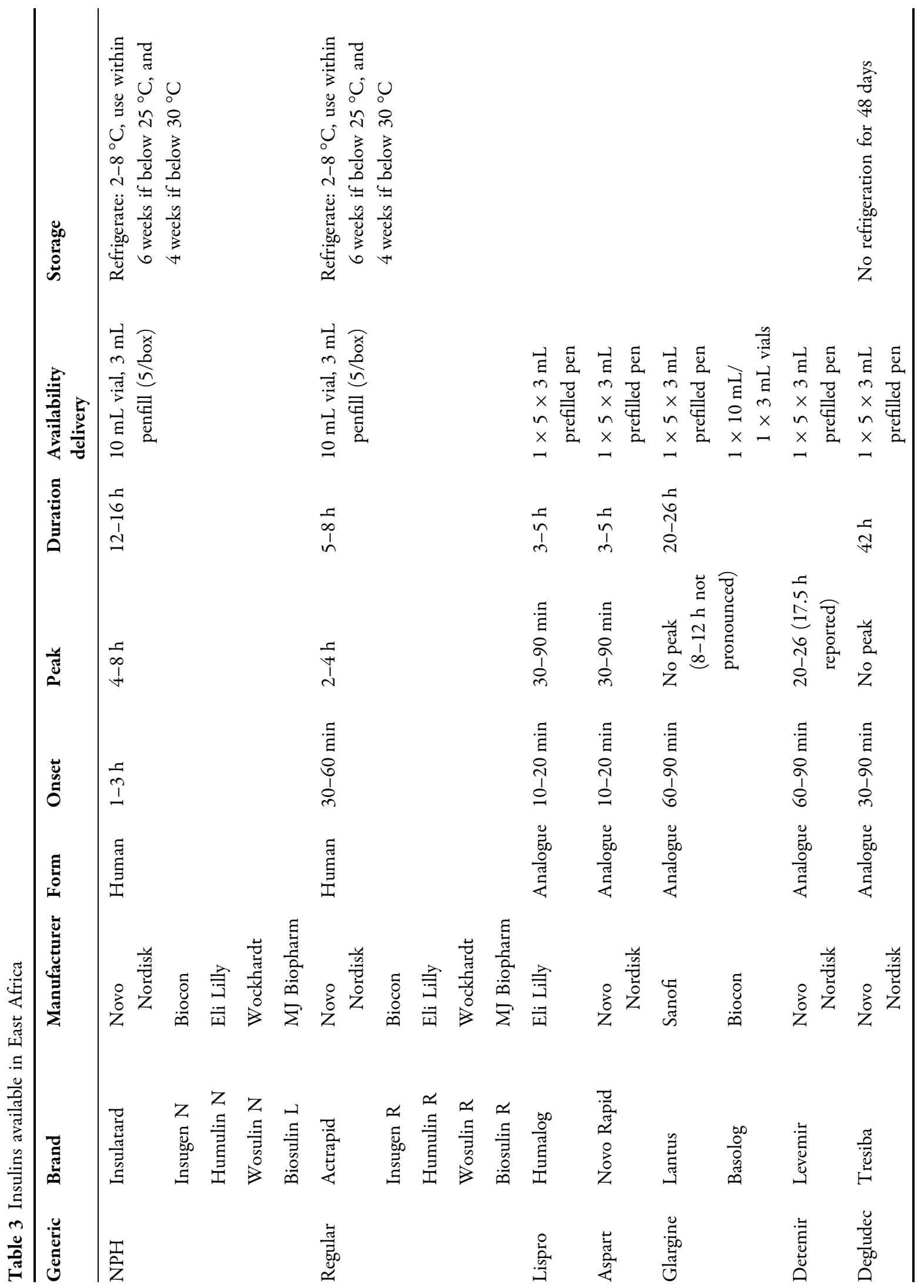




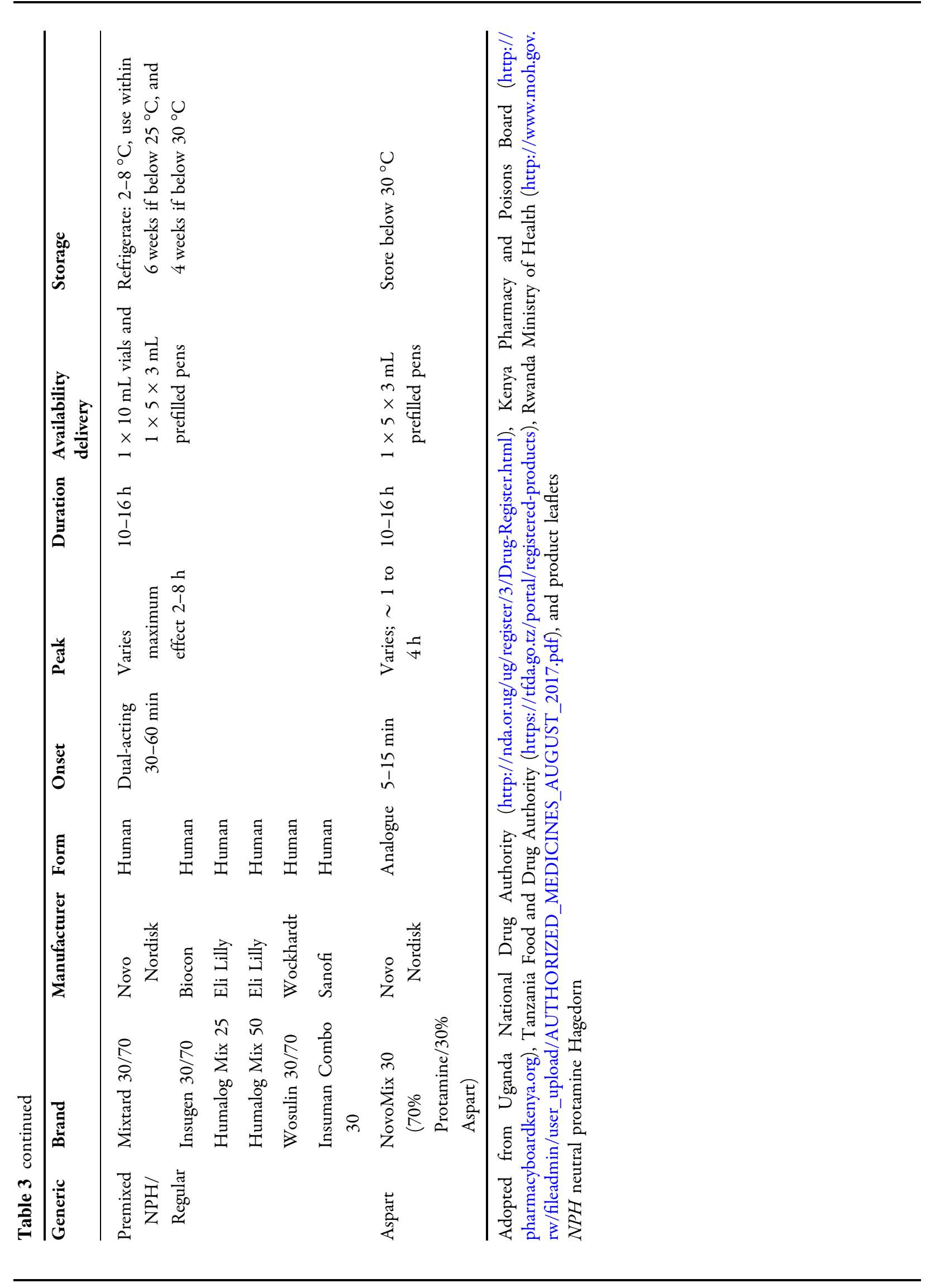




\section{Short-Acting Insulin}

Short-acting insulin is also commonly referred to as Regular or Neutral insulin. After a subcutaneous (SC) injection, the onset of action of Regular insulin is about 30-60 min, peak effect is in $2-4 \mathrm{~h}$, and duration of action is of $4-8 \mathrm{~h}$ $[47,63]$. The larger the dose of Regular insulin, the faster the onset of action, but the longer the time to peak effect and the longer the duration of the effect. U-100 Regular insulin is absorbed slightly more quickly than U-500. Regular insulin is metabolized in the liver, spleen, kidney, and muscle. The half-life is $30-60 \mathrm{~min}$ [64]. Without using other forms of insulin, shortacting insulin should be given every $6 \mathrm{~h}$, in four equal doses, without ever skipping a dose. Details of pharmacodynamics of short-acting insulin are displayed in Table 3.

\section{Rapid-Acting Insulin}

Rapid-acting insulins are typically insulin analogues that were developed to better control excursions of blood glucose following a meal ingestion, by achieving a pharmacokinetic profile more similar to mealtime endogenous insulin than human unmodified insulin does [65]. In East Africa the three commonly encountered rapid-acting insulin analogues are insulin lispro (Humalog ${ }^{\mathbb{B}}$; Eli Lilly, Indianapolis, IN, USA), insulin aspart (Novolog ${ }^{\circledR} /$ NovoR$\operatorname{apid}^{\circledR} ;$ Novo Nordisk, Bagsvaerd, Denmark), and insulin glulisine (Apindra $^{\circledR}$; Sanofi, Paris, France). Rapid-acting insulin should be injected before each meal in three daily doses if the patient is also taking a long-acting or intermediate-acting insulin, or six times a day if used without basal insulin. Rapid-acting insulins are only available in pharmacies located in the main towns of East Africa, and should be used taking into consideration the cost and the benefits gained when compared with human insulin that is cheaper and readily available.

\section{Basal (Long-Acting) Insulin}

Insulin is secreted at a low (basal) level in the non-fasted state, with increased, stimulated levels at mealtimes [49]. Several exogenous long-acting insulin formulations are now available in the market. In East Africa, the available long-acting insulins include neutral protamine Hagedorn (NPH), insulin glargine U-100, insulin glargine U-300, insulin detemir, and insulin degludec. They mainly vary in their duration of action and peak effect. The lower the peak, the lower the risk of hypoglycemia.

Compared with NPH insulin, insulin glargine shows a flatter pharmacologic profile with no pronounced peak and longer duration of action of about $24 \mathrm{~h}$. Within-subject variability has been shown to be lower with insulin glargine relative to NPH insulin [49]. Results from a meta-analysis of clinical trials show that among all the basal insulins, insulin degludec with ultra-long duration of action exhibits a greater reduction of $\mathrm{HbA1c}$, least variability of action, and lowest rate of hypoglycemia [66]. In persons prone to hypoglycemia, insulin degludec is the preferred basal insulin. However, the higher cost of this insulin and its unavailability in pharmacies outside main towns in East Africa make the recommendation for its use in the region to be highly individualized.

\section{Premixed Insulins}

Premixed insulins are short-acting insulin (Regular/Neutral) or rapid-acting analogue insulin mixed with intermediate-acting insulin in a fixed ratio, addressing both FPG and PPG in a single injection (biphasic human insulin [30/ $70,50 / 50]$, biphasic insulin aspart [30/70, 50/50], and biphasic insulin lispro [25/75, 50/50]). Those available in East Africa are shown in Table 3. The perceived advantages of using premixed insulin over a self-mixed insulin include the increased accuracy of dosage, efficacy, and patient convenience, which may translate to increased compliance and thus better long-term control of diabetes [67, 68].

\section{INSULIN THERAPY IN T1DM}

In the absence of obesity, all patients less than 30 years old should be treated as for T1DM unless they are less than 1 year old, have no 
ketones, have optic atrophy, retinitis pigmentosa, deafness, or other systemic illness [69]. T1DM is treated with insulin [70]. Treatment focuses on preventing complications by managing blood glucose levels with insulin, diet, and lifestyle modification [71, 72]. Multiple daily injections of short-acting or rapidacting insulin analogues, given $0-15$ min before meals together with one or more daily separate injections of intermediate or long-acting insulins, are used. The basal-bolus regimen includes basal insulin (insulin degludec, insulin glargine, insulin detemir, and $\mathrm{NPH}$ ) and bolus insulin (rapid-acting: insulin aspart, insulin lispro, or insulin glulisine; or short-acting: Regular/Neutral). Patients with severe decompensation (e.g., diabetic ketoacidosis, DKA) require intensive therapy, usually using short-acting insulin under close supervision [73]. In the DCCT study in which short-acting and intermediate-acting human insulins were used, intensive therapy with multiple daily injections or continuous subcutaneous insulin infusion (CSII) improved glycemia and resulted in better long-term outcomes [74].

The basal-bolus regimen showed improved PPG control and less hypoglycemia when compared with Regular insulin. Preprandial administration of insulin glulisine or insulin lispro showed better glycemic control [75]. Insulin aspart has been associated with improved quality of life (QOL). In patients with good glycemic control, insulin detemir and insulin glargine (with Regular insulin or bolus insulin) lowered FPG with less nocturnal hypoglycemia when compared with once- or twice-daily NPH insulin [76]. Improved QOL was reported in patients with use of insulin glargine when compared with use of NPH in a basal-bolus insulin regimen [77]. The preinjection hyperglycemia in T1DM with insulin glargine can be prevented by twice-daily administration of the insulin. Twice-daily insulin detemir in a basalbolus regimen showed less nocturnal hypoglycemia and improved glycemic control in several studies [78]. An ultra-long-acting insulin analogue, insulin degludec, in T1DM showed comparable safety and tolerability and less hypoglycemia when compared with insulin glargine [79]. As a result of their inflexible timing, long-acting analogue insulins may lead to hypoglycemia. The disadvantages of inflexibility with long-acting analogue insulin may be addressed with the use of modern insulin pump therapy. Evidence showed that premixed insulin analogues resulted in significant reduction in HbA1c levels [80] and similar safety profile [81] when compared with human premixed insulins. Moreover, premixed insulin analogues resulted in a better PPG control when compared with premixed human insulin.

Pramlintide, an amylin analogue, works by delaying gastric emptying, blunts pancreatic secretion of glucagon, and enhances satiety in T1DM. It has been shown to induce weight loss and lower insulin doses in T1DM. Pramlintide is not available in East Africa. For the management of T1DM in obese patients, the use of metformin reduces the insulin requirements and the total cholesterol/low-density lipoprotein (LDL) ratio with less weight gain [82].

\section{Recommendations}

- Basal-bolus insulin therapy is a standard of care in management of diabetes in T1DM (Grade A, EL I)

- For T1DM patients with minimal metabolic decompensation (minimal dehydration, fully conscious) initiation starts with initial dose ranging from 0.4 to 1.0 units/ kg/day (Grade A, EL III)

- Multiple daily injections of short-acting or rapid-acting insulin analogues given 0-15 min before meals together with one or more daily separate injections of intermediate or long-acting insulins are used. The basal-bolus regimen includes basal insulin (insulin degludec, insulin glargine, insulin detemir, and NPH) and bolus insulin (rapid-acting: insulin aspart, insulin lispro, or insulin glulisine; or shortacting: Regular/Neutral) (Grade A, EL I)

- Consider using rapid-acting insulin analogues for less hypoglycemia risk (Grade B, EL I)

- If other insulins are not available, premixed insulin injections may be used in T1DM adolescent patients. Premixed 
insulin analogues should be considered over human insulin for favorable degree of PPG control and significant lowering of HbA1c (Grade C, EL III)

- Patient education on matching prandial insulin doses to carbohydrate intake, premeal blood glucose levels, and anticipated physical activity should be encouraged (Grade A, EL II)

\section{TYPE 2 DIABETES MELLITUS (T2DM)}

T2DM is a progressive disease leading to oral hypoglycemic failure and subsequent requirement for insulin therapy. Therefore, the key concept in the treatment of T2DM is establishing individualized glycemic goals based on each patient's clinical characteristics. This individualized care influences the choice of antihyperglycemic therapy as the disease progresses over time.

Insulin is indicated in known patients with T2DM if the HbA1c level remains persistently above $10.0 \%(86 \mathrm{mmol} / \mathrm{mol})$ or uncontrolled diabetes with respect to predefined goals in spite of optimizing the oral antidiabetic drugs (OADs).

The initial step would be combining OADs with basal insulin (augmentation). If this intervention does not result in the required glycemic control, then consideration should be given to change the therapy to replacement therapy with insulin, which should be intensified as appropriate for the individual.

In newly diagnosed T2DM patients who are symptomatic, insulin may be the initial therapy to stabilize the glycemia and alleviate the symptoms (rescue therapy).

An algorithm showing the OADs leading to insulin is depicted in Fig. 3.

\section{Insulin Therapy in T2DM: The Initiation Algorithm}

Initiation of insulin therapy is preceded by a decision on the right insulin regimen, identifying the right formulation, doses, and appropriate delivery devices, and correct strategies for dose titration. The patient-centered treatment plan in the management of diabetes should focus specifically on matching the insulin supply to the regular diet/exercise patterns of diabetes patients and follow-up with regular SMBG $[10,83]$. The overall strategy is to first correct FPG with a dinnertime/bedtime insulin followed by a focus on PPG. As elevated PPG levels are a substantial contributor to daytime hyperglycemia, targeting PPG control becomes vital in achieving optimal glycemic control.

Once the decision has been made to initiate insulin, clinical, pharmacological, and psychosocial factors must be considered and factored into the patients care plan. In addition, other factors such as cost of insulin, quality, cold chain management for insulin, and continuous availability of insulin preparations as well as delivery devices should be contextually discussed with the patient, family, and other caregivers.

The FPG and PPG measurements together with $\mathrm{HbA1c}$ value provide some information for the physician to choose an insulin type by following simple ratios: ratio of prandial and FPG index ([PPG - FPG]/FPG); a high ratio implies a higher prandial component, which will require premixed or rapid-acting/short-acting insulin, while a low level suggests a greater contribution of the fasting component of hyperglycemia and supports the use of basal insulin. The ratio of FPG to HbA1c with a cutoff 1.3 gives an indication of the contribution of fasting hyperglycemia. Serum 1,5-anhydroglucitol (1,5-AG) drops as serum glucose rises above the renal threshold and has been proposed as a marker for postprandial hyperglycemia. In clinical practice HbA1c and 1,5-AG may be used sequentially, first utilizing HbA1c to identify patients who are moderately or well controlled (HbA1c 6.5-8.0\% [48-64 $\mathrm{mmol} / \mathrm{mol}]$ ) and then using the 1,5-AG assay to determine the extent of prandial glucose excursions [84]. Initiating insulin therapy with basal insulin is recommended by ADA 2018 and AACE/ACE [10, 17]. The IDF recommends to initiate insulin therapy with either basal or premix insulins [52]. The premix insulin analogues are preferred over human premix insulins owing to the lower incidence of severe hypoglycemia, less nocturnal hypoglycemia, and flexibility of administration [53]. The 
Patient newly diagnosed with type 2 Diabetes Mellitus

$\left(\mathrm{HbA}_{1 \mathrm{c}} \geq 86 \mathrm{mmol} / \mathrm{mol}\right.$; blood glucose level $\left.\geq 16.7 \mathrm{mmol} / \mathrm{L}\right)$

\section{$\downarrow$}

\section{Initiate Basal Insulin}

Usually with metformin $+/-$ other noninsulin agents

Initial Dose: $10 \mathrm{U} /$ day or $0.1-0.2 \mathrm{U} / \mathrm{Kg} /$ day

Adjustments: $10-15 \%$ or $2-4$ units once or twice weekly to reach FPG target

For hypoglycemia: Diagnose and address cause; if no clear reason, decrease dose by 4 units or $10-20 \%$

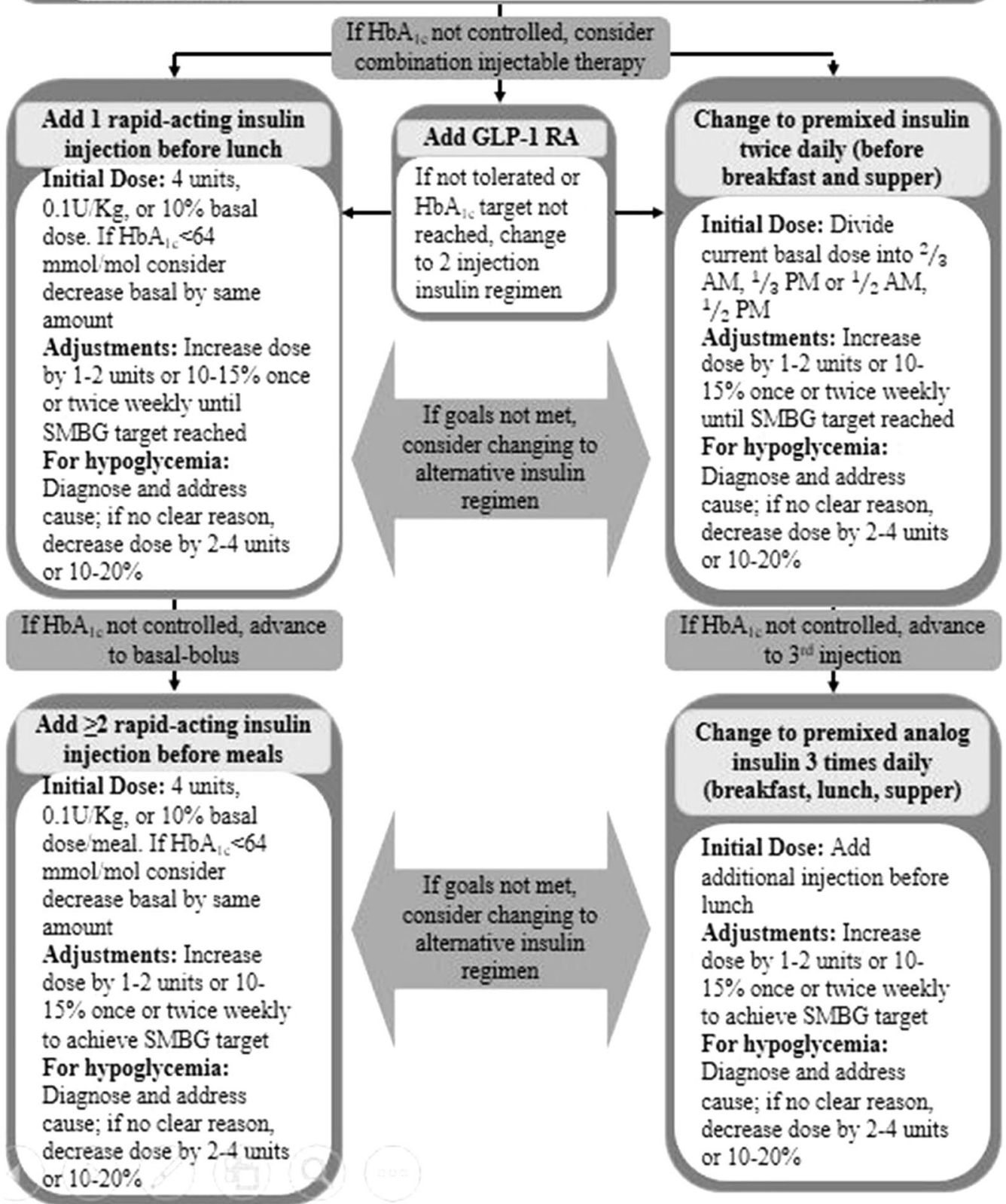

Fig. 3 Initiation of insulin therapy with basal insulin. HbAlc glycated hemoglobin Alc, FPG fasting plasma glucose, GLP-1 RA glucagon like peptide-1 receptor agonist, SMBG self-monitoring of blood glucose Modified from [104] 


\section{Lifestyle Modifications}

(Diet, weight management and adequate physical activity)

\section{Metformin}

Failure to reach $\mathrm{HbA}_{1 \mathrm{c}}$ target (e.g. $\mathrm{HbA}_{1 \varsigma}<53 \mathrm{mmol} / \mathrm{mol}$ ) after $>3 \mathrm{months}$ of Metformin monotherapy

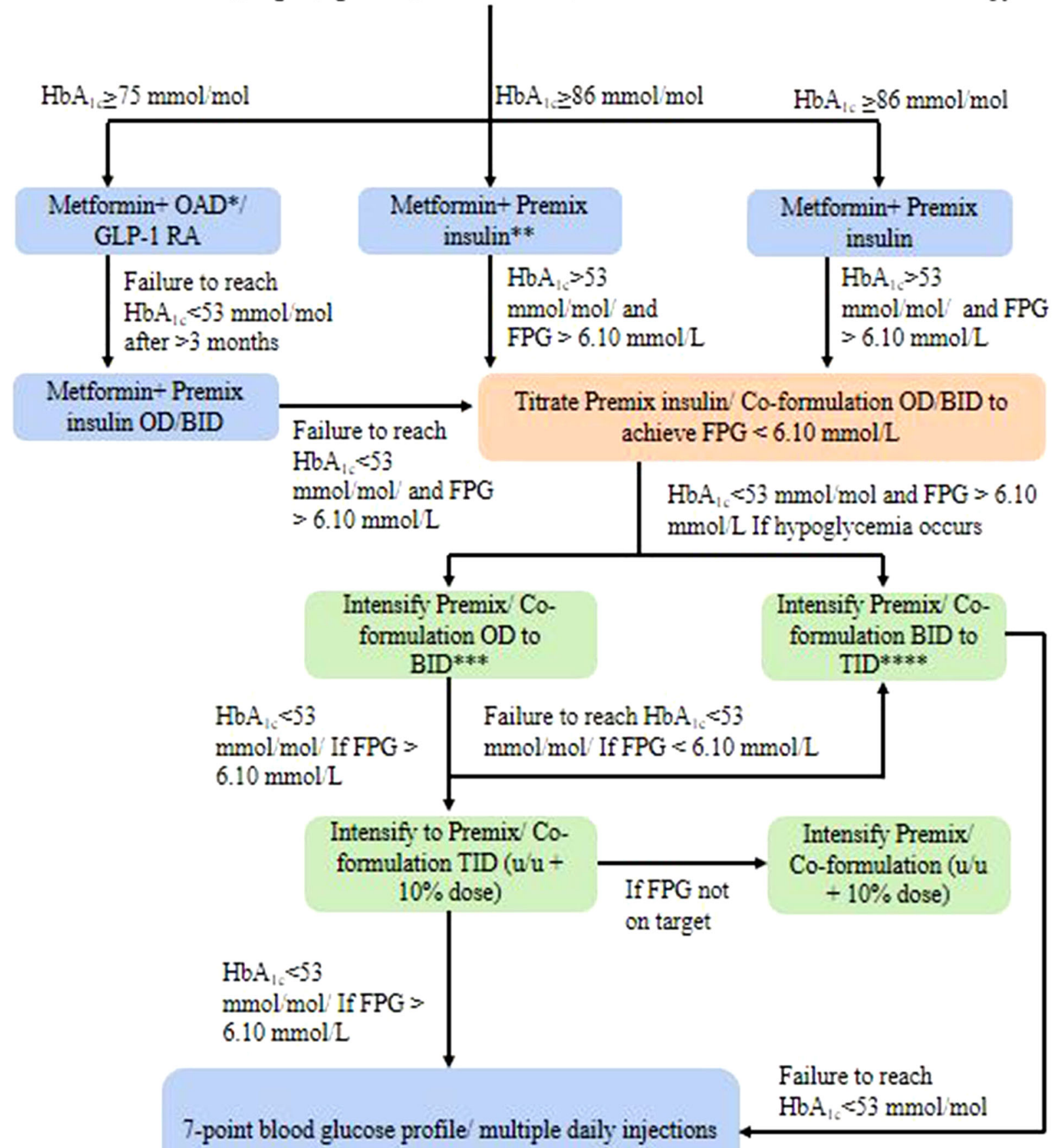


4Fig. 4 Initiation of insulin therapy with premix/insulin co-formulation. OAD oral antidiabetic agents, GLP-1 RA glucagon-like peptide-1 receptor agonist, OD once daily, BID twice daily, TID three times in a day. ${ }^{*} \mathrm{OAD}$ can be a sulfonylurea/thiazolidinedione/dipeptidyl peptidase-4 inhibitor or any other drug as per clinician's judgment; **Start with OD 10-12 units (0.1-0.2 U/kg body weight). In the morning if the predinner blood glucose is high. In the evening if the prebreakfast blood glucose is high. Split the dose when dose is $>30$ units. ${ }^{* * *}$ Intensification from OD to BID. Split the OD dose into equal breakfast and dinner doses (50:50). ${ }^{* * * *}$ Intensification from BID to TID. Add 2-6 U or $10 \%$ of total daily premix dose before lunch. Down-titration of morning dose $(-2$ to $4 \mathrm{U})$ may be needed after adding lunch dose. In both cases, continue metformin and administer premix just before meals Modified from [54]

Table 4 Comparisons between premixed human insulins vs premixed insulin analogues vs insulin co-formulation in patients with T2DM Modified from [54]

\begin{tabular}{llll}
\hline Parameter & $\begin{array}{l}\text { Premixed } \\
\text { human } \\
\text { insulin }\end{array}$ & $\begin{array}{l}\text { Premixed } \\
\text { insulin } \\
\text { analogue }\end{array}$ & $\begin{array}{l}\text { Insulin co- } \\
\text { formulation }\end{array}$ \\
\hline PPG control & + & +++ & +++ \\
FPG control & ++ & ++ & +++ \\
HbAlc control & + & ++ & ++ \\
Less & + & ++ & +++ \\
hypoglycemia & & & +++ \\
Mealtime & + & ++ & ++ \\
flexibility & & & ++ \\
Weight gain & + & + & + \\
\hline
\end{tabular}

$P P G$ postprandial glucose, $F P G$ fasting plasma glucose, HbAlc glycated hemoglobin Alc

Indian National Consensus Group (INCG) 2013 recommends only premix insulin at the initiation. In addition, as rescue therapy, the INCG recommends initiation of insulin in newly diagnosed T2DM patients [54].

\section{Basal (Long-Acting) Insulin Regimen}

Basal insulin controls glycemia by suppressing hepatic glucose production in between meals and during sleep. The intermediate-acting NPH, long-acting (insulin glargine and insulin detemir), or ultra-long-acting (insulin degludec) formulations offer relatively uniform 24-h coverage of blood glucose levels. Evidence showed that insulin glargine, insulin detemir, and insulin degludec are associated with less overnight hypoglycemia when compared with NPH and relatively less weight gain [85]. Several comparative trials between insulin glargine, insulin detemir, and insulin degludec show varying dose requirements for effective glycemic control, and higher average unit requirement with insulin detemir compared with insulin glargine [86, 87]. Figure 3 shows the steps for initiation with basal insulin therapy.

\section{Bolus Insulin Regimen}

Most patients with T2DM may require mealtime bolus insulin dosing in addition to basal insulin. Rapid-acting analogues (insulin aspart, insulin lispro, or insulin glulisine) are preferred owing to their prompt onset of action after dosing. The recommended starting dose of mealtime insulin is 4 units, 0.1 units $/ \mathrm{kg}$, or $10 \%$ of the basal dose.

\section{Premix Insulin Regimen}

Most patients with T2DM are treated with premix insulins (biphasic human insulin [30/70, 50/50], biphasic insulin aspart [30/70, 50/50], or biphasic insulin lispro $[25 / 75,50 / 50]$ ) or insulin degludec/insulin aspart 70/30. Figure 4 shows steps for initiating premix/insulin co-formulations. Premix insulin (10 U) once daily (OD) can be started either in the morning if predinner glucose is high or at night if the prebreakfast glucose is high. If a patient on biphasic insulin aspart 30 OD or BID has within-target FPG but has an $\mathrm{HbA} 1 \mathrm{c}>7.0 \% \quad(>53 \mathrm{mmol} / \mathrm{mol})$, a switch to biphasic insulin aspart 30 BID or TID should be considered. If their FPG is above target, the dose should be titrated to achieve FPG $4.0-6.0 \mathrm{mmol} / \mathrm{L}$; however, if hypoglycemia occurs, an additional daily dose should be added rather than further dose titration [88]. When the daily insulin dose in OD regimen exceeds $20 \mathrm{U}$, intensify the regimen to BID such 
that the dose is distributed as two-thirds in the morning and one-third in the evening. However, when the single dose exceeds $30 \mathrm{U}$, the dose can be split into two equal doses, which reduces the chance of hypoglycemia. Also, the initial dose distribution ratio for morning and evening doses is 50:50\% for biphasic insulin aspart 30, biphasic insulin lispro 25, and insulin degludec/insulin aspart 70/30 in case of patients with higher HbA1c or if blood glucose control is suboptimal [54]. The lower incidence of major and nocturnal hypoglycemia and flexibility of administration with premix insulin analogues have made this regimen a better choice over human premix insulins when initiating insulin therapy. However, insulin degludec/insulin aspart 70/30 may be preferred over other premix insulin analogues considering lower incidence of overall and nocturnal hypoglycemia and superior FPG control when used [53]. The advantages of premix insulin analogues and insulin co-formulations over premix human insulins are displayed in Table 4 [53].

Recent studies seem to suggest better outcomes with insulin analogues. Results from the A1chieve study showed that T2DM initiated with premix insulin was associated with a mean change of $\mathrm{HbA} 1 \mathrm{c}$ of $1.7 \%$ [- $18 \mathrm{mmol} / \mathrm{mol}]$ from a baseline of $9.1 \%[76 \mathrm{mmol} / \mathrm{mol}]$ and significantly reduced PPG in an African subgroup similar to those in the overall population [89]. In addition, switching from human insulin to premix analogues showed a similar positive safety profile. Evidence suggests that in insulinnaïve African subjects with T2DM, initiating once-daily premix insulin with or without OADs achieved better glycemic control than when compared with human insulin [90]. In a systematic review, short-term intensive insulin therapy was reported to improve the underlying pathophysiology in newly diagnosed T2DM. The study results showed that intensive insulin therapy leads to an increase in $\beta$-cell function and a decrease in insulin resistance when compared with baseline values [91].

In patients with T2DM poorly controlled on OADs with HbA1c $>9.0 \%$ (>75 mmol $/ \mathrm{mol}$ ), initiating premix insulin analogue therapy is superior to basal insulin analogues or human premix insulin [92-99]. The initiation of premix insulin analogues is recommended in insulinnaïve patients aged more than 65 years with T2DM poorly controlled by OADs with dietary counseling interventions for improved glycemic control and significant reduction in FPG.

\section{Recommendations}

Initiation

- Do not delay the decision to initiate insulin in diabetes patients (Grade A, EL I)

- Health care professionals (HCPs) should educate T2DM patients about insulin regimens and appropriate choice of regimen (Grade A, EL III)

- Consider initiating insulin therapy with or without metformin in patients with newly diagnosed T2DM who are symptomatic and/or have HbA1c $\geq 10.0 \%$ $(\geq 86 \mathrm{mmol} / \mathrm{mol}$ ) and/or blood glucose levels $\geq 16.7 \mathrm{mmol} / \mathrm{L}$ (Grade A, EL I)

- Initiate with once-daily basal insulin, once-daily premixed/co-formulation insulin, or twice-daily premixed insulin, either alone or in combination with GLP-1 RA (either alone or in combination with basal insulin, in same pen device) or in combination with other OADs on the basis of clinical features, glucose profile, risk of hypoglycemia, and patient preference (Grade A, EL II)

- Basal-bolus insulin regimens may be needed in severe hyperglycemia and in life-threatening or organ/limb-threatening clinical situations (Grade A, EL III)

- Analogue insulins with possible lower risk of nocturnal and symptomatic hypoglycemia may be used in preference to human insulins; however, economic considerations must be taken into account. Newer insulin co-formulations are associated with fewer hypoglycemic episodes (Grade B, EL I)

- Match the insulin dose to carbohydrate intake (Grade A, EL II)

- Counseling on scheduling regular blood glucose monitoring and awareness of hypoglycemic symptoms and their 
Table 5 Initiation of basal therapy Modified from [17]

\begin{tabular}{|c|c|c|}
\hline & Glucose value & Total daily dose \\
\hline \multirow[t]{2}{*}{ Step 1: initiation with basal insulin ${ }^{a}$} & $\mathrm{HbAlc}<8 \%(<64 \mathrm{mmol} / \mathrm{mol})$ & $0.1-0.2$ units $/ \mathrm{kg}$ \\
\hline & $\mathrm{HbAlc}>8 \%(>64 \mathrm{mmol} / \mathrm{mol})$ & $0.2-0.3$ units $/ \mathrm{kg}$ \\
\hline \multirow[t]{5}{*}{ Step 2: titration ${ }^{\mathrm{b}}$ (every $2-3$ days to reach glycemic goals) } & Fixed regimen & Increase by 2 units/day \\
\hline & Adjustable regimen & \\
\hline & $\mathrm{FPG}>10 \mathrm{mmol} / \mathrm{L}$ & Add 4 units \\
\hline & FPG $7.77-10 \mathrm{mmol} / \mathrm{L}$ & Add 2 units \\
\hline & FPG $6.11-7.72 \mathrm{mmol} / \mathrm{L}$ & Add 1 unit \\
\hline \multirow[t]{2}{*}{ Step 3: monitor for hypoglycemia } & $\mathrm{BG}<3.88 \mathrm{mmol} / \mathrm{L}$ & Reduce by $10-20 \%$ \\
\hline & $\mathrm{BG}<2.22 \mathrm{mmol} / \mathrm{L}$ & Reduce by $20-40 \%$ \\
\hline
\end{tabular}

HbAlc glycated hemoglobin A1c, $B G$ blood glucose, $F P G$ fasting plasma glucose, $N P H$ neutral protamine Hagedorn, $S U$ sulfonylureas

a Consider discontinuing SU therapy and basal analogues should be preferred over NPH insulin

b For most patients with T2DM taking insulin, glucose goals are HbA1c $<7 \%(<53 \mathrm{mmol} / \mathrm{mol})$ and fasting and premeal blood glucose $<6.11 \mathrm{mmol} / \mathrm{L}$ in the absence of hypoglycemia. HbAlc and FPG targets may be adjusted on the basis of patients age, duration of diabetes, presence of comorbidities, diabetic complications, and hypoglycemia risk

management are recommended to all patients initiating with insulin. The HCPs should provide guidance for adjusting insulin dose adjustments, administration, storage, and other practical aspects (Grade A, EL I)

Titration

- Target for FPG level is $4.4-7.2 \mathrm{mmol} / \mathrm{L}$, PPG level is $<10 \mathrm{mmol} / \mathrm{L}$, and 2-h PPG level is $<7.8 \mathrm{mmol} / \mathrm{L}$. These targets can be individualized on the basis of the risk of hypoglycemia and the urgency for glycemic control (Grade A, EL I)

- Titration should be done at regular and short intervals to attain glycemic goals without causing hypoglycemia (Grade A, EL I)

\section{Intensification of Insulin Therapy}

The long-term follow-up UKPDS and DCCT stressed the importance of intensive glycemic control with insulin, especially from the early stages of diagnosis of diabetes $[9,83]$.

\section{Basal Plus/Basal-Bolus Insulin}

As a result of progressively diminishing insulin secretory capacity, more patients with T2DM may require prandial insulin therapy in addition to the existing one or two doses of insulin. This is typically achieved with Regular insulin administered about $30 \mathrm{~min}$ before meals or rapid-acting insulin analogues such as insulin lispro, insulin aspart, or insulin glulisine, which can be injected just before or with the meal. Insulin analogues give better PPG control than human Regular insulin. Furthermore, an analogue-based basal-bolus regimen may be preferred over human basal-bolus regimen considering the significantly lower risk of nocturnal hypoglycemia and better outcomes in patients with T2DM [100]. The steps for initiation of basal therapy and intensification of insulin therapy are shown in Tables 5 and 6 , respectively.

\section{Premix Insulin}

The INCG 2013 recommends to intensify premix insulin to twice and thrice daily if HbA1c is $>7.0 \% \quad(>53 \mathrm{mmol} / \mathrm{mol}) \quad$ and $\quad$ FPG is $>6.1 \mathrm{mmol} / \mathrm{L}$ [54]. If glycemic control with 
Table 6 Intensification of premix/insulin co-formulation Modified from [17]

\begin{tabular}{|c|c|c|}
\hline & Therapeutic option & Total daily dose \\
\hline Step I: add prandial insulin & $\begin{array}{l}\text { When glycemic targets are } \\
\text { unmet }\end{array}$ & $\begin{array}{l}\text { TDD } 0.3-0.5 \text { units } / \mathrm{kg}(40-50 \% \text { basal: } 50-60 \% \\
\text { prandial })^{\mathrm{a}}\end{array}$ \\
\hline \multirow[t]{8}{*}{$\begin{array}{l}\text { Step II: titration }{ }^{\mathrm{b}} \text { (every } 2-3 \text { days } \\
\text { to reach glycemic goals) }\end{array}$} & $\begin{array}{l}\text { Fixed regimen (prandial } \\
\text { insulin) }\end{array}$ & Increase TDD by 2 units/day \\
\hline & \multicolumn{2}{|c|}{ Adjustable regimen (prandial insulin) } \\
\hline & $\mathrm{FPG}>9.99 \mathrm{mmol} / \mathrm{L}$ & Increase TDD by 4 units \\
\hline & FPG 7.77-9.99 mmol/L & Increase TDD by 2 units \\
\hline & FPG $6.10-7.71 \mathrm{mmol} / \mathrm{L}$ & Increase TDD by 1 unit \\
\hline & $\begin{array}{l}\text { 2-h PPG or next premeal } \\
\text { glucose }>9.99 \mathrm{mmol} / \mathrm{L}\end{array}$ & Increase prandial dose for the next meal by $10 \%$ \\
\hline & $\begin{array}{l}\text { When glycemic targets are } \\
\text { unmet }\end{array}$ & $\begin{array}{l}\text { TDD } 0.3-0.5 \text { units } / \mathrm{kg}(40-50 \% \text { basal: } 50-60 \% \\
\text { prandial)* }\end{array}$ \\
\hline & $\begin{array}{l}\text { FPG/premeal } \\
\mathrm{BG}>9.99 \mathrm{mmol} / \mathrm{L}\end{array}$ & Increase TDD by $10 \%$ \\
\hline \multirow{3}{*}{$\begin{array}{l}\text { Step III: monitor for } \\
\text { hypoglycemia }\end{array}$} & Fasting hypoglycemia & Reduce basal insulin dose \\
\hline & Nighttime hypoglycemia & $\begin{array}{l}\text { Reduce basal insulin or reduce short/rapid-acting } \\
\text { insulin taken before supper or evening snack }\end{array}$ \\
\hline & Between-meal hypoglycemia & Reduce previous premeal short/rapid-acting insulin \\
\hline
\end{tabular}

$B G$ blood glucose, DPP-4 dipeptidyl peptidase-4 inhibitors, $F P G$ fasting plasma glucose, $G L P-1$ glucagon-like peptide 1 receptor agonists, NPH neutral protamine Hagedorn, $P P G$ postprandial glucose, $S G L T 2$ sodium glucose cotransporter 2, $T D D$ total daily dose

a Basal + prandial insulin analogues preferred over $\mathrm{NPH}+$ Regular insulin or premixed insulin

b For most patients with T2DM taking insulin, glucose goals are $\mathrm{HbAlc}<7 \%(<53 \mathrm{mmol} / \mathrm{mol})$ and fasting and premeal blood glucose $<6.10 \mathrm{mmol} / \mathrm{L}$ in the absence of hypoglycemia. HbAlc and FPG targets may be adjusted on the basis of patient's age, duration of diabetes, presence of comorbidities, diabetic complications, and hypoglycemia risk

premix/basal insulin is not achieved then twicedaily insulin degludec/insulin aspart 70/30 is preferred over premix insulin analogues for intensification. Furthermore, a recent systematic review suggests that in insulin-treated T2DM, insulin degludec/insulin aspart 70/30 twice daily is comparable to biphasic insulin aspart 30 twice daily and imposes a lower risk of nocturnal hypoglycemia [101]. Insulin degludec/insulin aspart 70/30 improved long-term glycemic control with greater reduction in FPG with a lower dose and less nocturnal hypoglycemia, when compared with biphasic insulin aspart 30 [102-104].

\section{GLP-1 Receptor Agonists}

The injectable glucagon-like peptide-1 receptor agonists (GLP-1 RAs) (liraglutide, exenatide, lixisenatide, dulaglutide, and albiglutide) mimick the effects of endogenous GLP-1, thereby stimulating pancreatic insulin secretion in a glucose-dependent fashion, suppressing pancreatic glucagon output, slowing gastric emptying, and decreasing appetite. Advantages of this regimen include significant weight loss. However, this therapy produces nausea and vomiting, particularly early in the course of treatment. Generally, GLP-1 RAs and their combinations are not available in East Africa. 


\section{Combination Injectable Therapy (Insulin + GLP-1 RA)}

Consider a combination injectable therapy if the basal insulin has been titrated to acceptable FPG level or if the dose is $0.5 \mathrm{U} / \mathrm{kg} /$ day and HbA1c remains above the target. Advantages include less hypoglycemia risk and less weight gain. GLP-1 RAs are associated with transient gastrointestinal (GI) side effects.

\section{Recommendations}

Intensification

- Intensification of insulin therapy should be considered when patients fail to achieve glycemic goals even after optimal dose titration (Grade A, EL I)

- Intensification with premix insulin twice daily or thrice daily, insulin co-formulation-based regimen, prandial insulin (basal plus or basal bolus) with the largest meal of the day, or GLP-1 RA. Choice of intensification regimen is based upon diet, lifestyle, risk of hypoglycemia and weight gain, affordability, and patient preference (Grade A, EL II)

GLP1-RA therapy

- In T2DM patients with uncontrolled hyperglycemia, GLP-1 RAs are suitable second-line or third-line treatment option (Grade A, EL I)

\section{Insulin in Special Populations}

\section{Newly Diagnosed T2DM}

Insulin therapy (with or without additional agents) in newly diagnosed T2DM is preferred if HbA1c $\quad$ is $\geq 10.0 \% \quad(\geq 86 \mathrm{mmol} / \mathrm{mol})$, FPG $>13.9 \mathrm{mmol} / \mathrm{L}, \quad P P G>16.7 \mathrm{mmol} / \mathrm{L}$, and/ or if the patient is symptomatic. Consider initiating dual therapy in patients with newly diagnosed T2DM if $\mathrm{HbA} 1 \mathrm{c} \geq 9 \%$ ( $\geq 75 \mathrm{mmol} / \mathrm{mol}$ ) [10]. After glycemic and metabolic control, patients may be started on oral hypoglycemic agents.

\section{Recommendations}

- For newly diagnosed T2DM, consider initiating insulin therapy, if $\mathrm{HbA} 1 \mathrm{c} \geq 10.0 \%$ $(\geq 86 \mathrm{mmol} / \mathrm{mol}), \quad$ FPG $>13.9 \mathrm{mmol} / \mathrm{L}$, PPG $>16.7 \mathrm{mmol} / \mathrm{L}$, and/or if patient is symptomatic (Grade A, EL I)

- For newly diagnosed T2DM, consider initiating dual therapy, if HbA1c 9.0\% $(\geq 75 \mathrm{mmol} / \mathrm{mol})$ (Grade A, EL II)

\section{Elderly}

Elderly patients with diabetes are at an increased risk of hypoglycemia and therapy with a low risk of hypoglycemia should be the choice of treatment. Metformin is the first-line agent for older adults with T2DM. Use of SUs and other insulin secretagogues with high risk of hypoglycemia should be used with caution. When insulin therapy is required, most elderly patients with advanced diabetes complications, life-limiting coexisting chronic illnesses, or limited functional status, once-daily basal insulin injection therapy is preferred to multiple daily injections as the latter may be too complex for them. SMBG using home glucose meters is encouraged; patients or their caregivers are instructed on dose adjustment according to results of SMBG and steps to take for hypo- and hyperglycemic episodes [10].

\section{Recommendations}

- Consider antihyperglycemic therapy with low risk of hypoglycemia in elderly patients who are at increased risk of hypoglycemia (Grade A, EL II)

- Consider once-daily basal insulin injection regimen over multiple daily insulin injection regimen to reduce the risk of hypoglycemia if glycemic goals can be achieved within the individualized HbA1c target (Grade B, EL II)

\section{Pregnancy}

Pregnancy outcomes of mothers with diabetes during pregnancy are associated with high rates of complications in both the mother and baby. Polyhydramnios, intrauterine fetal death, macrosomia, and stillbirths are frequently reported [105-107]. Glycemic targets during pregnancy have become more stringent [108]: the HbA1c goal is $6.0-6.5 \%$ 
(42-48 mmol $/ \mathrm{mol}$ )-the goal of $6.0 \%$ ( $42 \mathrm{mmol} / \mathrm{mol}$ ) may be optimal if this can be achieved without significant hypoglycemia, but the target may be relaxed to $7.0 \%$ (53 mmol/mol) if necessary to prevent hypoglycemia; FPG $5.3 \mathrm{mmol} / \mathrm{L}$; PPG $7.8 \mathrm{mmol} / \mathrm{L}$; and 2-h PPG $6.7 \mathrm{mmol} / \mathrm{L}$. Where glycemic targets have been achieved with metformin monotherapy, the use of metformin during pregnancy has been associated with a lower risk of neonatal hypoglycemia and less maternal weight gain than insulin. In the short term, in women with gestational diabetes mellitus (GDM) requiring drug treatment, glibenclamide is clearly inferior to both insulin and metformin, while metformin (plus insulin when required) performs better than insulin [109]. In most East African countries, insulin (between once and three times daily) is the treatment of choice to control hyperglycemia in GDM. Recent data suggest no significant difference between premix insulin analogues and premix human insulin in terms of glycemic control or fetal outcome (neonatal birth weight). However, premix insulin analogues offer flexible dosing and a high safety profile compared with premix human insulin [110].

\section{Recommendations}

- Tighter glycemic targets are suggested during pregnancy: HbA1c 6.0-6.5\% (42-$48 \mathrm{mmol} / \mathrm{mol}$ ); FPG $5.3 \mathrm{mmol} / \mathrm{L}$; $1-\mathrm{h} \mathrm{PPG}$ $7.8 \mathrm{mmol} / \mathrm{L}$; and 2-h PPG $6.7 \mathrm{mmol} / \mathrm{L}$ (Grade A, EL I)

- Insulin is the preferred medication for treating hyperglycemia in gestational diabetes mellitus (GDM) as it does not cross the placenta (Grade A, EL I)

- Antenatal patients may use any human insulin preparation, insulin aspart or insulin lispro preparations, or insulin detemir (Grade A, EL II)

\section{Recommendation}

- Consider using premix insulin with proven safety profile in lactating mothers with diabetes (Grade B, EL II)

\section{Lactation}

Individualized treatment approach is advised in lactating mothers with diabetes. It is safe to treat them with premix insulin owing to their proven safety profile.

\section{Renal Impairment}

The overall prevalence of chronic kidney disease (CKD) in sub-Saharan Africa (SSA) is $13.9 \%$ with a wide variation between East African regions (from 30\% in Zimbabwe to 2\% in Côte d'Ivoire) [111]. Chronic renal failure (CRF) is associated with diverse alterations in carbohydrate and insulin metabolism. Insulin therapy with premix insulin analogues can notably improve glycemic control in CRF diabetic patients [112]; however, the choice of insulin therapy should be individualized.

\section{Recommendations}

- Consider using insulin analogues in renal impaired patients with diabetes for improved glycemic control with low risk of hypoglycemia (Grade B, EL II)

- Frequent blood glucose monitoring and dose adjustments are recommended in chronic renal failure (CRF) diabetic patients when they are treated with insulin (Grade B, EL II)

\section{Cardiac Impairment}

Coronary heart disease (CHD) affects $5-8 \%$ of diabetic patients in SSA [113]. Premix insulin analogues reduce PPG more effectively than premix human insulins and basal insulin analogues. The choice of insulin regimen should be individualized and based upon cost, severity of hyperglycemia, risk of hypoglycemia, and likelihood of interventional procedures in the very near future.

\section{Recommendations}

- Choice of insulin regimen and preparation should be based upon cost, severity of hyperglycemia, risk of hypoglycemia, and likelihood of interventional procedure in near future (Grade B, EL II)

- Patients with T2DM on combination therapy of premixed insulin analogues and 
OADs should be carefully monitored for signs and symptoms of heart failure (HF), weight gain and edema, and a prompt clinical action is recommended if any deterioration in cardiac symptoms occurs (Grade B, EL II)

\section{Hepatic Impairment}

The patients with T2DM are at risk of developing non-alcoholic fatty liver disease (NAFLD) and therefore patients with diabetes and hepatic impairment are likely to be encountered in East Africa [114]. When insulin therapy is required in patients with hepatic impairment, the choice should be regimens with low risk of hypoglycemia.

\section{Recommendation}

- Consider using insulin analogues in T2DM patients with hepatic impairment for improved glycemic control with low risk of hypoglycemia (Grade B, EL II)

\section{Monogenic Forms of Diabetes}

Mutations which may be dominantly or recessively inherited from either parent or occur as a de novo mutation in a single gene affect the functioning of the insulin-producing pancreatic $\beta$-cells and precipitate a rare form of diabetes termed as monogenic. The worldwide prevalence of the monogenic form of diabetes is estimated at $1-2 \%$ of all pediatric diabetes [115]. Nyangabyaki-Twesigye et al. reported the first case in East Africa with permanent neonatal diabetes due to a mutation in the KCNJ11 gene encoding the Kir6.2 subunits in a 6-month-old subject [116]. The monogenic form of diabetes can be differentiated as transient and permanent neonatal diabetes. In both conditions, hyperglycemia is a common phenomenon. In the transient type, the patient may recover spontaneously at 3 months with no further requirement for insulin. The International Society for Pediatric and Adolescent Diabetes (ISPAD) recommends genetic testing for diagnosis of this rare form of diabetes for optimal treatment [117]. Although such tests are expensive and not readily available in East
Africa, some centers outside East Africa offer them, for research purposes. In most patients with permanent neonatal diabetes, lifelong insulin therapy is required, and management follows guidelines for T1DM [118]. In children with an ATP-sensitive potassium channel defect in the pancreatic $\beta$-cells, treatment with oral high dose glibenclamide is an optional therapy.

\section{Recommendation}

- In most patients with permanent neonatal diabetes, lifelong insulin therapy is required (Grade A, EL III)

\section{Ramadan and Other Fasting States}

Many patients with diabetes, T1DM and T2DM, will fast during religious fasts. The Epidemiology of Diabetes and Ramadan (EPIDIAR) study showed that $42.8 \%$ of T1DM subjects and $78.7 \%$ of T2DM subjects fasted for at least 15 days during Ramadan [119]. The CREED study reported that $94.2 \%$ of T2DM subjects fasted for at least 15 days and $63.6 \%$ fasted every day [120]. When fasting, insulin resistance/deficiency can lead to excessive glycogen breakdown and increased gluconeogenesis. This poses a risk of hypoglycemia, hyperglycemia, ketoacidosis, dehydration, and thrombosis [121]. Insulin therapy in religious fasting requires that the patient is educated on the risks posed by fasting, is familiar with SMBG, adheres to appropriate nutrition intake, proper exercise, and dose adjustment to minimize complications [122]. East Africa is lacking in studies to describe the characteristics and multiple approaches to the management of people with diabetes who fast during Ramadan and other religious fasts. Premix insulin analogues have proven efficacy and safety profile with lower rates of hypoglycemia and hence are preferred over premix human insulins in patients with insulin therapy during religious fasting periods [123-126]. Insulin glargine has been safely used in fasting Muslim T2DM patients [127]. Insulin detemir given at $40 \%$ of the daily dose at predawn meal (suhoor) and 60\% as biphasic insulin aspart 30 at sunset (iftar) showed non-inferiority when compared with standard care without fasting $[128,129]$. Figure 5 gives the recommended insulin adjustments and dose 
titrations based on SMBG in diabetes, young adults/adolescents with T1DM, and pregnant women during Ramadan. If a patient is taking $\mathrm{NPH}$ or premix insulin at suhoor, it is important to check blood glucose at noon before up-titration of the pre-suhoor dose. If noon blood glucose is $<6.1 \mathrm{mmol} / \mathrm{L}$ and pre-iftar blood glucose is not at target, a long-acting insulin analogue may be preferred. For those on insulin and SU, a decision on the need to reduce doses of both agents or to start with insulin only is required on the basis of individual assessment. Use of insulin lispro and insulin pumps was reported to be safe in fasting T1DM [130, 131].

\section{Religious Fasting Recommendations}

- T2DM patients wishing to fast and who are on premix insulin analogue therapy are recommended to use the usual morning dose at sunset (iftar) and half the usual evening dose at predawn (suhoor) meal, e.g., patients on biphasic insulin aspart 30 with 30 units in morning and 20 units in the evening before Ramadan, the recommended dose will be 30 units in the evening (iftar) and 10 units in the morning (suhoor) during Ramadan (Grade A, EL II)

- In T2DM patients with NPH or premix human insulin at suhoor, it is recommended to check blood glucose at noon before up-titration of the pre-suhoor dose (Grade B, EL II)

- Before starting Ramadan or other religious fasts, consider educating the patient with diabetes on risk quantification, blood glucose monitoring, nutrition intake, proper exercise, and dose adjustments to minimize hypoglycemic complications (Grade A, EL III)

- Consider advising pregnant women during Ramadan who are on sulfonyl urea

\section{Switch to insulin analogue}

\section{Long- or intermediate-acting basal insulin}

Rapid- or short-acting prandial/bolus insulin

Premixed insulin
- OD - NPH*/detemir/glargine/degludec. Take at iftar. Reduce dose by $15-30 \%$ - $\mathrm{BID}-\mathrm{NPH} / \mathrm{detemir} /$ glargine. Take usual morning dose at ifar. Reduce evening dose by $50 \%$ and take at suhoor

-Take normal dose at iftar. Omit lunch-time dose. Reduce suhoor dose by $25-50 \%$

-OD - Take normal dose at iftar

-BID - Take usual morning dose at iftar. Reduce evening dose by $25-50 \%$ and take at suhoor -TID - Omit afternoon dose. Adjust iftar and suhoor doses

Dose titration should be performed every three days and dose adjustment made according to BG level

\begin{tabular}{l|l|l|l|}
\hline $\begin{array}{l}\text { Fasting/ Pre-iftar/ Pre- } \\
\text { suhoor BG }\end{array}$ & \multicolumn{1}{|c|}{ Pre-iftar** } & \multicolumn{2}{|c|}{ Post-iftar**/ Post-suhoor*** } \\
\cline { 2 - 4 } & Basal insulin & Short-acting insulin & Premixed insulin \\
\hline$<3.9 \mathrm{mmol} / \mathrm{L}$ or symptoms & $\begin{array}{l}\text { Reduce by } 4 \text { units } \\
\text { Reduce by } 2 \text { units }\end{array}$ & $\begin{array}{l}\text { Reduce by } 4 \text { units } \\
\text { Reduce by } 2 \text { units }\end{array}$ & $\begin{array}{l}\text { Reduce by } 4 \text { units } \\
\text { Reduce by } 2 \text { units }\end{array}$ \\
\hline $.0-5.0 \mathrm{mmol} / \mathrm{L}$ & No change required & No change required & No change required \\
\hline $5.1-7.0 \mathrm{mmol} / \mathrm{L}$ & Increase by 2 units & Increase by 2 units & Increase by 2 units \\
$7.1-11.1 \mathrm{mmol} / \mathrm{L}$ & Increase by 4 units & Increase by 4 units & Increase by 4 units \\
$>11.1 \mathrm{mmol} / \mathrm{L}$ & &
\end{tabular}

Fig. 5 Insulin adjustments and dose titrations in fasting young adults/adolescents with T1DM, and pregnant women during Ramadan. BG blood glucose, BID twice daily, NPH neutral protamine Hagedorn, OD once daily,
TID three times a day. ${ }^{*}$ Alternatively, reduced NPH dose can be taken at suhoor or at night; ${ }^{* *}$ adjust the insulin dose taken before suboor; ${ }^{* * *}$ adjust the insulin dose taken before iftar Adopted from [122] 
Table 7 Glycemic management in patients with acute febrile illness (AFI) Adapted from [134]

\begin{tabular}{|c|c|}
\hline & Glycemic management \\
\hline $\begin{array}{l}\text { AFI patients with adequate oral } \\
\text { intake }\end{array}$ & $\begin{array}{l}\text { Frequent BGM to check for hyperglycemic episodes } \\
\text { Continue OADs in patients eating well if } \mathrm{BG} \text { is well controlled and no } \\
\text { contraindication with OADs } \\
\text { Initiate insulin If BG is poorly controlled with OADs }\end{array}$ \\
\hline $\begin{array}{l}\text { AFI patients with compromised oral } \\
\text { intake }\end{array}$ & Modification in diet (small portion sizes, at frequent intervals) \\
\hline $\begin{array}{l}\text { AFI patients on concomitant } \\
\text { corticosteroid therapy }\end{array}$ & $\begin{array}{l}\text { In steroid-induced or worsened hyperglycemia, subcutaneous insulin using a basal or } \\
\text { multiple daily injections regimen }\end{array}$ \\
\hline $\begin{array}{l}\text { AFI patients with compromised } \\
\text { hepatorenal function }\end{array}$ & Rapid-acting insulin in small, frequent doses to manage hyperglycemia \\
\hline $\begin{array}{l}\text { AFI patients with compromised } \\
\text { sensorium }\end{array}$ & $\begin{array}{l}\text { Discontinue OADs and initiate IV insulin } \\
\text { Alternatively, SC rapid-acting insulin may be used }\end{array}$ \\
\hline AFI in elderly patients & Frequent BGM to detect atypical symptoms of hyperglycemia and hypoglycemia \\
\hline AFI patients with cachexia/asthenia & $\begin{array}{l}\text { An insulin regimen which provides both prandial and basal coverage, such as } \\
\text { premixed/dual action or basal plus/basal-bolus insulin in patients with lack of } \\
\text { energy (asthenia), with or without wasting, loss of weight, muscle atrophy, fatigue, } \\
\text { and loss of appetite (cachexia) during the febrile or convalescence phase }\end{array}$ \\
\hline
\end{tabular}

$O A D$ oral antihyperglycemic drugs, $B G M$ blood glucose monitoring, $A F I$ acute febrile illness, $I V$ intravenous, $I M$ intramuscular, $S C$ subcutaneous

therapy and/or insulin to exercise caution because of the high risk of hypoglycemia (Grade D, EL IV)

\section{Infections}

Malaria and other acute febrile illnesses (AFI) are frequent causes of fever in patients with diabetes who reside in East Africa. Hyperglycemia may follow any AFI but equally hypoglycemia has been encountered in malaria and sepsis [132, 133]. In addition, hyperglycemia in patients with AFI may be secondary to medications administered (e.g., steroids). Quinine administration has been associated with hypoglycemia [133]. The glycemic management in patients with AFI should take into consideration compromised GI absorption, renal, hepatic, and cognitive dysfunctions, asthenia, and/or cachexia in such patients [134]. The overall goal is to avoid hypoglycemia [135] and to minimize glycemic variability.
Table 7 displays the glycemic management in patients with AFI.

\section{Recommendations}

- Consider using subcutaneous (SC) insulin with a basal or multiple daily injections regimen in steroid-induced or worsened hyperglycemia (Grade B, EL III)

- Consider using Regular insulin or rapidacting insulin in small, frequent doses to manage hyperglycemia in acute febrile illness patients with metabolic decompensation, compromised hepatorenal function, or cognitive impairment (Grade B, EL III)

- Consider using premixed or basal plus/ basal-bolus insulin in patients with lack of energy (asthenia), with or without wasting, loss of weight, muscle atrophy, fatigue, and loss of appetite (cachexia) during 
Table 8 Management of diabetes in patients with HIV Adapted from [137]

\begin{tabular}{|c|c|}
\hline Strategies & Management \\
\hline $\begin{array}{l}\text { General } \\
\text { management }\end{array}$ & $\begin{array}{l}\text { Treatment for comorbid conditions } \\
\text { Hypertension-treatment with ACE inhibitors and ARBs not an optimal choice in patients with } \\
\text { HIV } \\
\text { Dyslipidemia - treatment with pravastatin, fluvastatin, atorvastatin, and rosuvastatin in patients with } \\
\text { HIV }\end{array}$ \\
\hline $\begin{array}{c}\text { Non-insulin } \\
\text { therapies }\end{array}$ & $\begin{array}{l}\text { Use metformin if well tolerated and if no contraindications are present } \\
\text { Use SU/alpha-glucosidase inhibitors if metformin is contraindicated/not tolerated. Thiazolidinediones } \\
\text { and DPP-4 inhibitors are also used in patients with HIV } \\
\text { Use incretin mimetics if weight loss is desired }\end{array}$ \\
\hline Insulin & $\begin{array}{l}\text { Initiate basal-bolus regimen or premixed insulin }(1.0 \mathrm{U} / \mathrm{kg} / \mathrm{day}) \text { at diagnosis } \\
\text { Insulin may be tapered or reduced }(0.5 \mathrm{U} / \mathrm{kg} / \text { day }) \text { once control is achieved } \\
\text { Initiate insulin aspart in patients with ketonuria and for critically ill patients } \\
\text { Educate HIV-infected patients on how to dispose of lancets, glucose strips, insulin syringes, pens, and } \\
\text { needles to prevent HIV transmission }\end{array}$ \\
\hline $\begin{array}{c}\text { Changes in } \\
\text { HAART }\end{array}$ & $\begin{array}{l}\text { Pre-existing T2DM may continue to be managed after diagnosis of HIV by continuing with the same } \\
\text { drug therapy that was being used prior to detection of HIV } \\
\text { Patients diagnosed with diabetes and HIV together may be treated with metformin if well tolerated and } \\
\text { if no contraindications are present. Depending on the baseline HbAlc, insulin or low dose } \\
\text { meglitinides can be initiated as a second-line therapy } \\
\text { Patients developing diabetes after HAART may be treated with metformin or other OADs. Insulin is a } \\
\text { better and safer choice and may be tapered or reduced once control is achieved }\end{array}$ \\
\hline
\end{tabular}

$A C E$ angiotensin converting enzyme, $A R B$ angiotensin receptor blockers, $H I V$ human immunodeficiency virus, $H C W$ health care worker, $S U$ sulfonylurea, $D P P-4$ inhibitors dipeptidyl peptidase-4 inhibitors, $H A A R T$ highly active antiretroviral therapy

the febrile or convalescence phase (Grade B, EL III)

\section{HIV Infection and Comorbidities}

An increased prevalence of hyperglycemia, insulin resistance, diabetic dyslipidemia, and lipodystrophy has been reported in diabetes patients with HIV infection [136]. Majority of patients presenting with comorbid diabetes and hyperglycemic may be managed as T2DM, taking into consideration comorbidity of infections [137]. Table 8 shows different strategies in the management of diabetes with HIV comorbidity.

\section{Recommendation}

- When insulin therapy is required, consider initiating insulin therapy with basalbolus regimen or premixed insulin at diagnosis. Higher doses may be needed for control (Grade C, EL III)

\section{Ketosis-Prone Diabetes}

Aggressive diabetes management in patients with ketosis-prone diabetes significantly improves $\beta$-cell function and insulin sensitivity 
often allowing the discontinuation of insulin therapy within a few months of initiation of the treatment. The period of near-normoglycemic remission may last for a few months to several years [138, 139].

\section{Recommendation}

- Initiate insulin as per the clinical situation, but keep a close watch for hypoglycemia. Sudden, significant downtitration of dose frequency and/or requirement may be needed (Grade $\mathbf{C}, \mathbf{E L}$ III)

\section{Self-Monitoring of Blood Glucose (SMBG)}

SMBG in diabetes management helps in making treatment decisions and correcting insulin dose. For optimal use of SMBG, regular review and interpretation by both the patients and physicians is required. In those individuals injecting insulin more than two times per day, SMBG should be done at least three times per day [140]. However, there is insufficient evidence on the number of times SMBG needs to be done in individuals on once-daily insulin therapy, with or without OADs [141]. Evidence from East Africa on the adherence to SMBG has shown suboptimal glycemic control, especially among those who had to pay for glucose strips. For optimal diabetes care in the region, to achieve adherence to SMBG and optimal glycemic control, it has been suggested that patient education be given alongside free glucose strips [142]. Once-daily testing (preferably in the morning) should be done to assess the efficacy of the basal insulin dose. When HbA1c is $>7.0 \%$ ( $>53 \mathrm{mmol} / \mathrm{mol}$ ) in spite of a satisfactory FPG, a second test should be performed after the largest meal of the day to exclude postprandial hyperglycemia. In a low-resource setting, it may be considered not cost-effective to perform a postprandial test if the HbA1c is at target [141].

\section{Recommendation}

- Pragmatic suggestions for SMBG should accompany insulin prescriptions (Grade D, EL IV)

- Basal insulin is best monitored by FPG, 1-2 times a week (Grade A, EL II)

- Prandial insulin is best monitored by paired premeal and postmeal glucose values (Grade A, EL II)

- Premixed insulin is initially monitored by prebreakfast and predinner glucose values, followed by postprandial values (Grade A, EL 2)

\section{Exercise}

Exercise increases insulin sensitivity, and blood glucose should be checked before and after exercise and appropriate action taken [10].

\section{Recommendation}

- Check blood glucose before and after exercise, and take appropriate action. If exercise is enduring, e.g., bicycle riding, check blood glucose in between exercise and take appropriate action (Grade A LE I)

\section{HYPOGLYCEMIA, WEIGHT GAIN, AND PSYCHOSOCIAL ASPECTS}

\section{Hypoglycemia}

Medications that are associated with the highest risk of injury when used in error are known as high-alert medications. Insulin has long been identified as belonging to this group of medications [143] and patients should be appropriately educated on its use. Hypoglycemia has a significant negative impact on a person's wellbeing and QOL and can therefore influence adherence, compliance, and ultimately the success of the insulin therapy. In East Africa, surveys on hypoglycemia indicated high frequency episodes (T1DM, 88\%; T2DM, 69\%) with almost half of the study population being 


\section{Pre-operative}

- Pre-operative assessment and optimization of BGL (target BGL 5-10, $\mathrm{HbA} \leq c^{<7.0 \%)}$

- Usual dose of insulin and oral AHG on the day prior to surgery

- Fast from midnight

- Patients should not drive themselves to hospital

- Should be the first case on the morming list

- Omit morning dose of insulin (as well as all oral AHG) and cease insulin pump

- Commence I/D infusion before induction of anesthetics (before $10 \mathrm{am}$ )

- Patients on insulin pump will stop the pump

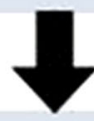

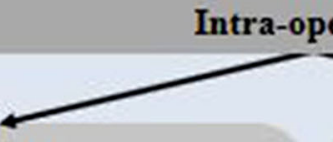

Major surgery

\section{when $I G$ infusion is started \\ peri-operative period}

\begin{tabular}{|c|c|c|c|}
\hline \multicolumn{4}{|c|}{ Post-operative } \\
\hline \multicolumn{2}{|c|}{$\begin{array}{l}\text { Major surgery } \\
\text { - Continue I G infusion and monitor BGL } \\
\text { hourly } \\
\text { - Restart s/c insulin / oral AHG / insulin } \\
\text { pump when patients able to tolerate solids } \\
\text { - Restart Metformin after } 24 \mathrm{hrs} \text { if } \\
\text { hemodynamically stable and serum } \\
\text { creatinine level normal post-procedure }\end{array}$} & \multicolumn{2}{|c|}{$\begin{array}{l}\text { Minor surgery } \\
\text { - Monitor BGL hourly in the post-operative } \\
\text { ward } \\
\text { For day-only surgery, consider admitting } \\
\text { patients if their BGL very erratic or their } \\
\text { oral intake inadequate post-procedure } \\
\text { - Restar usual } \mathrm{s} / \mathrm{c} \text { insulin / oral AHG } \\
\text { including Metformin with next meal }\end{array}$} \\
\hline Insulin regimen & \multicolumn{2}{|c|}{ Day of surgery } & Comments \\
\hline insulin pump & \multicolumn{2}{|l|}{ No change } & $\begin{array}{l}\text { Use 'sick day' or sleep basal rates for outpatient } \\
\text { surgery. discortinue if plamned admission }\end{array}$ \\
\hline Long acting, pesk less insulins & \multicolumn{2}{|c|}{$75-100 \%$ of moming dose } & $\begin{array}{l}\text { Recuce right time dose if history of noctumal or } \\
\text { moming hypoglycemia } \\
\text { On the day of surgery, the moming dose of basal } \\
\text { insulin may be sdministered on arnval to facility }\end{array}$ \\
\hline Intermediate-2cting insulins & \multicolumn{2}{|c|}{$50-75 \%$ of moming dose } & See conments for long-acting invulins \\
\hline Fired combination insulins & \multicolumn{2}{|c|}{$\begin{array}{l}50-75 \% \text { of morming dose of } \\
\text { intermediate acting component }\end{array}$} & $\begin{array}{l}\text { Lispro-protamine only available in combination: } \\
\text { therefore, use NPH instezd on dsy of surgery: See } \\
\text { comments for long-acting insulins }\end{array}$ \\
\hline Short- and rapid-zcting insulins & \multicolumn{2}{|l|}{ Hold the dose } & \\
\hline Non-inaulin injectable & \multicolumn{2}{|l|}{ Hold the cose } & \\
\hline
\end{tabular}


4Fig. 6 Perioperative management in T1DM and T2DM patients. I/G insulin-glucose infusion, AHG antihyperglycemic agents, BGL blood glucose level. *Includes patients with T1DM as well as insulin-requiring T2DM. Adopted from perioperative diabetes management guidelines, published on the Australian Clinical Practice Guidelines website (https://www.clinicalguidelines.gov.au)

unaware of the hypoglycemia [144]. Efforts to avoid hypoglycemia include patient education, SMBG, and improving insulin delivery through proper delivery devices and techniques.

\section{Weight Gain}

Insulin therapy is associated with increase in body weight [145]. Patients on insulin therapy should have appropriate physical activity and insulin dose adjustment tailored to carbohydrate intake to avoid excessive weight gain.

\section{Psychosocial Aspects}

Psychosocial barriers to successful insulin therapy in East Africa include lack of physician-patient interaction, understanding of diabetes and its treatments by both physicians and patients, and proper provision of testing and follow-up of patients [146].

\section{Educational Motivation/Counseling Support}

The patients should be educated on monitoring of glucose, insulin injection technique, insulin storage, recognition/treatment of hypoglycemia, and sick day management by qualified health educators.

\section{Mental Illness}

Evidence on the association between poor glycemic control and comorbid depression among T2DM patients in East Africa has shown that most T2DM patients with poor glycemic control witnessed further worsening of glycemic control with increasing depression. Insulin-treated T2DM patients with poor glycemic control should be screened for comorbid depression and provided with suitable interventions for optimized diabetes management [147].

\section{SPECIAL SITUATIONS}

In East Africa, for hospitalized patients with diabetes, hyperglycemia and hypoglycemia are associated with prolonged hospitalization, rehospitalization, increased morbidity, and high mortality [29]. Efforts should therefore be made in hospitalized patients with diabetes to achieve glycemic targets and prevent both hyperglycemia and hypoglycemia.

\section{Inpatient Settings}

Hyperglycemia in hospitalized patients is defined as blood glucose levels of $>7.8 \mathrm{mmol} / \mathrm{L}$ [148]. Alterations in diet or changes in antihyperglycemic treatment are warranted when blood glucose levels are persistently above $7.8 \mathrm{mmol} / \mathrm{L}$. If an $\mathrm{HbA} 1 \mathrm{c}$ from the previous 3 months is unavailable, measuring the HbA1c in all patients with diabetes or hyperglycemia admitted to the hospital is recommended. On admission, $\quad H b A 1 c \quad$ value $\geq 6.5 \%$ $(\geq 48 \mathrm{mmol} / \mathrm{mol}$ ) implies that diabetes preceded hospitalization, taking into consideration that the patient does not have a hemoglobinopathy, recent episode of malaria, bleeding, or hemolysis [148]. Blood glucose value of $3.9 \mathrm{mmol} / \mathrm{L}$ in hospitalized patients indicates hypoglycemia, whereas a blood glucose value of $3.0 \mathrm{mmol} / \mathrm{L}$ denotes clinically significant hypoglycemia [149]. Severe hypoglycemia is defined as that associated with severe cognitive impairment regardless of blood glucose level [149].

For the majority of critically ill patients and non-critically ill patients, at a blood glucose value of $10.0 \mathrm{mmol} / \mathrm{L}$ insulin therapy should be initiated for treatment of persistent hyperglycemia with the aim of achieving target glucose level in the range between 7.8 and $10.0 \mathrm{mmol} / \mathrm{L}$. More stringent goals (blood glucose values between 6.1 and $7.8 \mathrm{mmol} / \mathrm{L}$ ) in selected populations in the inpatient setting may occasionally be required and extreme caution taken to avoid hypoglycemia.

Blood glucose monitoring in patients who are eating should be performed before meals, while in patients who are not eating it should 
be performed every 4-6 h [148]. For patients on IV insulin, blood glucose monitoring should be done every $30 \mathrm{~min}$ to $2 \mathrm{~h}$.

\section{Treatment for Non-critically Ill Patients}

Insulin is the preferred treatment for glycemic control in critically ill patients [148]. For noncritically ill patients with good nutritional intake basal, meal-related, and correction insulin dose are preferred. Subcutaneous rapid- or short-acting insulin before meals or every $4-6 \mathrm{~h}$ may be used in patients not on regular meals or in patients receiving continuous enteral/parenteral nutrition to correct hyperglycemia [148]. For non-critically ill patients with poor oral intake or those who are taking nothing by mouth (NPO), basal insulin or a basal plus bolus correction insulin regimen is preferred. Basalbolus is preferred over sliding scale insulin (SSI) owing to improved glycemic control and reduced hospital complications. Premixed insulin is preferred in the outpatient setting [150] and basal-bolus therapy in the inpatient setting [151].

\section{Recommendations}

- Consider using basal plus bolus correction insulin regimen, with the addition of meal-related insulin in patients who have good nutritional intake, in non-critically ill patients (Grade A, EL I)

- Avoid sliding scale insulin in the inpatient hospital setting (Grade A, EL I)

\section{Treatment for Critically Ill Patients}

In the critically ill patients, continuous IV insulin is preferred. When the patient is able to take regular meals, basal and correction insulin doses are administered. When transitioning T1DM or T2DM patients to outpatient, SC insulin, SC basal insulin should be started $2-4 \mathrm{~h}$ before the IV insulin is discontinued.

\section{Surgery}

\section{Perioperative Management}

Perioperative management of blood glucose levels is based on the following objectives: (1) reduction in morbidity and mortality, (2) prevention of severe hyperglycemia or hypoglycemia, (3) maintenance of physiological electrolyte and fluid balance, (4) prevention of ketoacidosis, and (5) achieving the target glycemic levels less than $10 \mathrm{mmol} / \mathrm{L}$ in critical patients $[152,153]$ and less than $7.7 \mathrm{mmol} / \mathrm{L}$ in stable patients [148]. Long-acting insulin (insulin glargine) should be discontinued 2-3 days prior to surgery and combination of intermediate-acting insulin (NPH) with short- or rapidacting insulin twice daily or Regular insulin before meals and intermediate-acting insulin at bedtime used for glycemic control. Figure 6 shows the perioperative management in T1DM and T2DM patients.

\section{Patients on Enteral/Parenteral Nutrition}

Three doses of insulin such as basal, meal-related, and correctional insulins are administered in patients on continuous/bolus enteral or parenteral nutrition [154]. In patients on continuous enteral feedings, the basal insulin dose is based on the patient's preadmission basal insulin dose or $30-50 \%$ of the total daily dose (TDD) of insulin or 5 units of $\mathrm{NPH} /$ insulin detemir subcutaneously every $12 \mathrm{~h}$ or 10 units of insulin glargine every $24 \mathrm{~h}$. The dose of mealrelated insulin is calculated as 1 unit of insulin for every $10-15 \mathrm{~g}$ carbohydrate per day administered as Regular insulin every $6 \mathrm{~h}$ or rapidacting insulin every $4 \mathrm{~h}$. Correctional insulin should also be administered SC every $6 \mathrm{~h}$ using human regular insulin or every $4 \mathrm{~h}$ using a rapid-acting insulin such as insulin lispro, insulin aspart, or insulin glulisine. For patients receiving enteral bolus feedings, approximately 1 unit of Regular human insulin or rapid-acting insulin per $10-15 \mathrm{~g}$ carbohydrate should be given SC before each feeding. Correctional insulin coverage should be added as needed before each feeding. For patients receiving parenteral nutrition, Regular insulin may be added to the solution, particularly if 20 units of correctional insulin have been required in the past $24 \mathrm{~h}$. A starting dose of 1 unit of human regular insulin for every $10 \mathrm{~g}$ carbohydrate is recommended, to be adjusted daily in the solution. Correctional insulin should be administered SC. 
Table 9 Insulin dosing for enteral/parenteral feedings Adapted from [10]

\begin{tabular}{|c|c|c|}
\hline Situation & Basal/nutritional & Correctional \\
\hline $\begin{array}{l}\text { Continuous } \\
\text { enteral } \\
\text { feedings }\end{array}$ & $\begin{array}{l}\text { Continue prior basal or, if none, calculate from TDD or consider } \\
5 \text { units NPH/detemir every } 12 \mathrm{~h} \text { or } 10 \text { units glargine/degludec } \\
\text { daily nutritional: regular insulin every } 6 \mathrm{~h} \text { or rapid-acting insulin } \\
\text { every } 4 \mathrm{~h} \text {, starting with } 1 \text { unit per } 10-15 \mathrm{~g} \text { of carbohydrate; } \\
\text { adjust daily }\end{array}$ & $\begin{array}{l}\text { SC regular insulin every } 6 \mathrm{~h} \text { or rapid- } \\
\text { acting insulin every } 4 \mathrm{~h} \text { for } \\
\text { hyperglycemia }\end{array}$ \\
\hline $\begin{array}{l}\text { Bolus enteral } \\
\text { feedings }\end{array}$ & $\begin{array}{l}\text { Continue prior basal or, if none, calculate from TDD or consider } \\
5 \text { units NPH/detemir every } 12 \mathrm{~h} \text { or } 10 \text { units glargine/degludec } \\
\text { daily nutritional: give regular insulin or rapid-acting insulin SQ } \\
\text { before each feeding, starting with } 1 \text { unit per } 10-15 \mathrm{~g} \text { of } \\
\text { carbohydrate; adjust daily }\end{array}$ & $\begin{array}{l}\text { SC regular insulin every } 6 \mathrm{~h} \text { or rapid- } \\
\text { acting insulin every } 4 \mathrm{~h} \text { for } \\
\text { hyperglycemia }\end{array}$ \\
\hline $\begin{array}{r}\text { Parenteral } \\
\text { feedings }\end{array}$ & $\begin{array}{l}\text { Add regular insulin to TPN IV solution, starting with } 1 \text { unit per } \\
10 \mathrm{~g} \text { of carbohydrate; adjust daily }\end{array}$ & $\begin{array}{l}\text { SC regular insulin every } 6 \mathrm{~h} \text { or rapid- } \\
\text { acting insulin every } 4 \mathrm{~h} \text { for } \\
\text { hyperglycemia }\end{array}$ \\
\hline
\end{tabular}

$I V$ intravenous, $S C$ subcutaneous, $T D D$ total daily dose, $T P N$ total parenteral nutrition, $N P H$ neutral protamine Hagedorn

Table 9 shows insulin dosing for enteral/parenteral nutrition.

\section{Insulin Therapy in Patients in Their Home Setting}

The patients are usually stabilized on the target blood glucose levels before discharge from hospital. Management of insulin therapy while the patient is at home includes education about insulin regimens, appropriate choice of regimen, scheduling regular blood glucose monitoring, awareness on hypoglycemic symptoms and their management, contact details of the health care worker in the nearest health care facility in case of emergency, and a regular follow-up within 10-14 days' time.

\section{PRACTICAL ASPECTS}

\section{Insulin Delivery Devices}

Insulin can be administered via various methods such as vial and syringe, insulin pen, jet injectors, and continuous subcutaneous insulin infusion (CSII) using insulin pumps. Only inhaled insulin (Afrezza) is not injectable [155]. In East Africa, vial and syringe, insulin pens, and insulin pumps are accessible. The choice of insulin delivery device should be individualized for patients.

\section{Recommendation}

- Health care authorities and planners should be alerted to the risks associated with syringe or pen needles $6 \mathrm{~mm}$ or longer in children (Grade A, LE II)

\section{Insulin Transport and Storage}

Specific storage conditions provided by the manufacturer in the package inserts (stored for 28 days at $30{ }^{\circ} \mathrm{C} ; 45$ days at $25^{\circ} \mathrm{C}$ ) should be followed. Insulin should be stored in a cool $\left(<30^{\circ} \mathrm{C}\right)$ environment and must be protected from extremes of temperature such as direct sunlight, kitchen, glove box of a car, over the engine in a motor vehicle, or left in a closed stationary motor vehicle.

Insulin can be safely transported from the health facility or pharmacy in a bag that will not be exposed to excessive high temperatures. 
If exposure to high temperatures is envisaged, it is advised to transport the insulin in the original packaging placed on an icepack.

\section{Recommendation}

- Transportation of insulin from a health facility to home can be safely done without an icepack if no extremes of temperatures are envisaged. If uncertainty exists about an exposure to high temperatures $\left(>30^{\circ} \mathrm{C}\right)$ it is advised to transport insulin on an ice pack (Grade A, LE IV)

Lack of refrigeration facilities at home may force patients to use improvised cooling systems such as storing insulin vials submerged in water or keeping insulin in a pot with sand [156]. These should be used with caution as they may result in contamination of insulin and subsequent injection abscesses. Figure 2 shows insulin tattoos following the injection of contaminated insulin stored in a water container. The patient also overslanted the needle. When a reliable refrigerator is not available, it is advisable to follow the manufacture's advice in the package inserts and store insulin in a clean container at room temperature, in a clean environment (a cupboard and not under a bed), until more studies are available on insulin storage. Figure 7

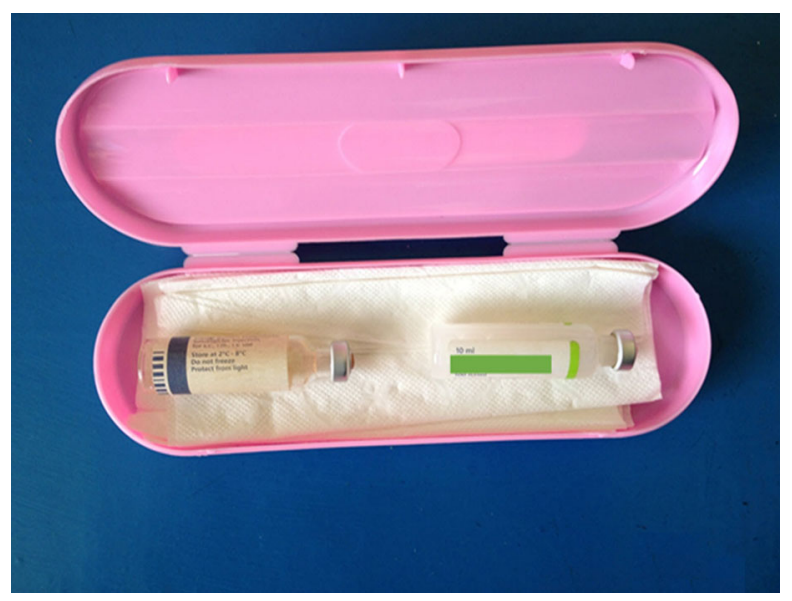

Fig. 7 Clean boxes used to safely keep insulin and insulin syringes at home Image courtesy of Silver Bahendeka shows such a container where insulin can be safely kept in a refrigerator or at room temperature in a cupboard.

\section{Injection Sites}

Correct technique in insulin delivery is critical for optimal control of diabetes. Various factors are considered while injecting insulin, including choice of site, injection site rotation, skin fold, and injection techniques. The recommended injection and infusion sites are the abdomen, thigh, buttock, and upper arm [157].

\section{Recommendation}

- Recommended injection and infusion sites are the abdomen, thigh, buttock, and upper arm (Grade A, LE I)

\section{Needle Length}

A 4-mm needle is long enough to traverse the skin and enter the SC tissue, with little risk of IM or intradermal injection. Therefore, it is considered the safest needle for adults and children regardless of age, sex, ethnicity, or body mass index (BMI). A 5-mm needle may be acceptable in obese individuals. Very young children ( $\leq 6$ years old) and very thin adults should use the $4-\mathrm{mm}$ needle by lifting a skin fold and inserting the needle perpendicularly into it. Others may inject using the 4-mm needle without lifting a skinfold. Previously needle lengths that were recommended for SC injections were $\geq 8 \mathrm{~mm}$ for adults and $\geq 6 \mathrm{~mm}$ for children. These are now considered to be too long because they increase the risk of IM injections without evidence of improved control [157]. The 4-mm needle should be inserted perpendicular to the skin, at $90^{\circ}$ to the skin surface, not at an angle, regardless of whether a skinfold is raised. Currently only the $6-\mathrm{mm}$ and larger syringe needles are available in East Africa. Raising the skin fold is therefore recommended until the 4-mm needles are available. 


\section{Recommendation}

- A 4-mm needle is recommended for all patients with diabetes (Grade A, LE I)

- The safest currently available syringe needle for all patients is $6 \mathrm{~mm}$ in length. However, when any syringe needle is used in children $\geq 6$ years old, adolescents, or slim to normal weight adults (body mass index of $19-25 \mathrm{~kg} / \mathrm{m}^{2}$-calculated as the weight in kilograms divided by the height in meters squared) injections should always be given into a lifted skin fold at $90^{\circ}$ (Grade A, LE I)

\section{Injection Site Complications}

Injection site complications include injection site infection and abscesses, injection site skin scarification (insulin tattoos), and lipohypertrophy. Local skin injection site complications are common in patients on insulin therapy in East Africa, because of poor insulin storage (keeping insulin in water to keep it cool), and wrong injection technique (overslanting the needle resulting in insulin being injected intradermally). Lipohypertrophy is a thick soft to firm swelling with rubbery consistency, which appears on the surface of the skin at the site of insulin injection. Injection sites should be rotated.

\section{Recommendation}

- Injections should be systematically rotated to avoid lipohypertrophy. This means at least $1 \mathrm{~cm}$ (1-finger breadth) from previous injections (Grade A, LE II)

- Injection sites should be examined by the health care worker at least once a year and preferably every visit (Grade A, LE II)

- After the insulin has been pushed in, patients should count slowly to 10 and then withdraw the needle from the skin. This is necessary to prevent medication leakage so as to get the full dose (Grade A, LE I)

- Many patients in East Africa reuse syringes for various reasons, including financial. This is not recommended by the manufacturer. There is an association between needle reuse and lipohypertrophy. Patients who reuse needles should not be subjected to alarming claims of excessive morbidity from this practice (Grade A, LE III)

\section{Needle Stick Injuries}

Needle stick injuries are common among physicians and health care workers and warrant training on preventive methods. Health care workers in Africa suffer 2-4 needle-stick injuries per year on average, with Nigeria and Tanzania reporting 2.10 injuries per health care worker on average [158], and this should be avoided.

\section{Recommendation}

- Safety injection devices should be considered first-line choice if injections are given by a third party. Pens and syringes with needles used in this setting should have protective mechanisms for all sharp ends of the delivery device (Grade A, LE II)

- The health care worker should be involved in the training of the patient and safe disposal of sharps (Grade A, LE I)

\section{Lipoatrophy}

Lipoatrophy is clinically characterized by visible cutaneous depression and palpable atrophy of SC fat tissue at the injection site. It is an immunological response to insulin aggregates in the presence of high circulating titers of antiinsulin autoantibodies. Rotation of injection sites minimizes this complication.

\section{Pain}

Pain is the commonest adverse event associated with insulin use. This is especially so with wrong injection technique and reuse of needles and syringes.

\section{Barriers and Myths Concerning Insulin}

Educating and training patients on diabetes selfmanagement can address patient-related barriers to insulin use. Barriers affecting physicians and health care workers could be addressed 
through programs such as skill enhancement and conferences. In addition, drug or devicespecific barriers can be addressed through continuing education programs, flexible insulin regimens, preparations, and modern devices.

\section{BIOSIMILAR INSULINS}

For many decades, the major sources for (animal) insulin were pancreata of pigs and cows. After discovery of the primary structure of the insulin molecule, insulin was manufactured by recombinant technology. This paved the way for pharmaceutical companies other than the original multinational ones to also manufacture and market similar insulins. Differences in the manufacturing processes (none of the insulin manufacturing procedures are identical) result in the fact all insulins that might become biosimilar differ from the originator insulin to some extent [159]. The current unanswered question is: do such differences in the structure of the insulin molecule and/or the purity and so on have clinically relevant consequences? The European Union guidelines for market approval require that the manufacturer demonstrate that the insulin has a safety and efficacy profile that is similar to that of the original insulin formulation. Biosimilar insulins are available on the East African market. Transferring from one insulin another requires a dose titration. There is no published data on the consequences of switching from one insulin to another in patients on insulin therapy in East Africa. However, observations from diabetes clinics indicate a need for titrating dosages at every switch.

\section{Recommendation}

- In switching from one insulin to another similar insulin (original to biosimilar) it is advised to carry out a dose titration always starting with a reduced dose and to uptitrate to avoid hypoglycemia. Self-monitoring of blood glucose is therefore necessary (Grade D, LE V)

\section{BENEFITS OF EARLY OPTIMIZED CONTROL OF DIABETES}

\section{Economic Consideration}

The high negative impact of diabetes on the economy of individuals, families, societies, and countries has been well established [2]. The direct costs relate to the cost of treatment of disease and related complications, while the indirect costs are associated with income losses through reduced productivity and disability. The present data on per patient expenditures for diabetes in SSA are extrapolated from the western world data. Accordingly, direct cost estimated per patient expenditure for diabetes in 2015 in SSA ranged between US\$243 and US\$419 [1]. A recent study data from LMIC showed average per patient costs of US\$580 [160]. The percentage of indirect costs for the East African region was at 39.7\% [161]. Diabetes is associated with catastrophic family expenditure; patients, family, and other care takers have to pay out-of-pocket to meet the expenses related to diabetes management [30]. While public health facilities in East Africa offer insulin for free, the supply is usually insufficient or the distance travelled to access the free insulin is more costly than the cost of insulin in the private sector. Insulin syringes are usually supplied in quantities that would be insufficient for single use. Glucose meters for SMBG are not available from the public health sector. These areas are of public concern and need to be urgently addressed by the health authorities as they adversely affect the outcome of diabetes management.

\section{Hit Early Hit Hard Paradigm Shift}

The conventional stepwise therapy approach in T2DM involves contributing factors like abnormal gastric emptying, insulin resistance, dysfunctional lipid metabolism, excess hepatic glucose production, $\beta$-cell failure, poor $\alpha$-cell regulation, and altered role of the kidney in handling glucose being addressed one-by-one in a sequential fashion. Current data suggests that the stepwise therapy approach to achieve 
glycemia, blood pressure, and lipid targets, despite significant improvements in the glycemic control and lipids, is associated with disappointingly low percentages of subjects attaining all three targets. This irregular pattern in glycemic control is due to lower control rates for all three targets [162]. Furthermore, this approach may lead to a decline in $\beta$-cell function.

Moreover, clinical inertia in the stepwise approach therapy may expose T2DM patients to prolonged periods of hyperglycemia of even up to 8 years [163]. Intervals between adding or switching agents were previously much longer than currently recommended by the ADA/EASD and AACE $[17,104]$. There still exists a difference of opinion between ADA/EASD and AACE guidelines on the management of T2DM in patients with HbA1c level of 7.5-9.0\% (58-$75 \mathrm{mmol} / \mathrm{mol}$ ) at diagnosis with regard to insulin therapy versus the addition sulfonyl urea. The 6-year-long Outcome Reduction with Initial Glargine Intervention (ORIGIN) study showed that at diagnosis of T2DM, early initiation of insulin in combination with OADs resulted in a stable pattern of glycemic control [164]. Several other studies have reported the beneficial effects on $\beta$-cell functions in patients with T2DM who were on early and aggressive treatment with insulin in combination with OADs [165-167].

Currently there are no studies that show the comparative efficacy of early initiation of insulin in combination with OADs versus stepwise addition of therapy over time. However, longerterm follow-up observational study in UKPDS [83] showed that better outcomes are possible by initiating intensive glycemic control in early stages of diabetes, preferably from the start of the diagnosis of diabetes. While there is still a need for more long-term studies, it appears that hit early hit hard with use of multiple therapies, including insulin, may alter the natural history of $\beta$-cell function in patients with T2DM.

\section{RESEARCH AGENDA}

The World Health Organization's STEPwise approach to surveillance (STEPS) data showed that there is a high rate of undetected diabetes in the East African region [23-28]. The high rates of undetected diabetes imply a likelihood of late presentation with complications in a health system already overburdened by high prevalence of infectious diseases, including HIV/AIDS, TB, and malaria. Overall, there is increased risk of adverse outcomes in diabetes patients in the East African region. Moreover, there are significant challenges in accessing diagnosis and treatment for diabetes, complicated by social, cultural, and ethnic factors.

This guideline recommends safely achieving tight glucose control by lifestyle modifications and achieving target values for HbA1c, FPG, PPG, hypoglycemia, nocturnal hypoglycemia, and glycemic variability in patients with diabetes, for optimized diabetes management. The guideline recommends intensive diabetes therapy in T1DM with basal-bolus (basal prandial) regimens that mimic the normal $\beta$-cell insulin secretion. For the treatment management in T2DM, the guideline recommends individualized glycemic goals based on FPG, PPG, and HbA1c levels. In addition, the guideline recommends initiation with once-daily basal insulin, once-daily premixed/co-formulation insulin, or twice-daily premixed insulin, either alone or in combination with GLP-1 RA (where available) or in combination with other OADs to achieve the glycemic goals and prevent longterm complications. The guideline has identified key concepts in optimal glycemic control in T2DM that included choice of an appropriate insulin regimen and stepwise approach of insulin initiation, titration, and intensification. The strength of the guideline is that the recommendations put forth are based on the existing established guidelines and published evidence. East Africa lacks sufficient evidence from RCTs and even data from small studies to support the use of insulin in special populations such as diabetes in pregnancy; diabetes and lactation; diabetes and renal, cardiac, and hepatic impairment; monogenic diabetes; and management in special situations like Ramadan and other faith-based fasting and acute and chronic infections. Therefore, the EADSG Guidelines Development Task Force resorted to an evidence-based consensus to arrive at the 
guidelines for use of insulin in such special populations and special situations.

The lack of reliable data on insulin use in the region necessitates the need for high-quality studies to be carried out in East Africa; they will help in making treatment decisions in diabetes management.

We hope that the current guidelines will be a useful reference tool to all health care professionals in East Africa and will lead to an improvement in the optimal care of diabetes in the region. These guidelines will be updated with respect to newer evidence and newer insulin formulations that will be available on the East African market in the near future and based on further observational research, involving large numbers of physicians and in the setting of routine outpatient care of diabetes in the Eastern Africa region.

\section{ACKNOWLEDGEMENTS}

Funding. No funding or sponsorship was received for this study or publication of this article.

Editorial Assistance. Editorial assistance in the preparation of this article was provided by Balajee Narayanan of Cognizant Technology Solutions India Pvt Ltd. Support for this assistance was funded by Novo Nordisk.

Authorship. All named authors meet the International Committee of Medical Journal Editors (ICMJE) criteria for authorship for this article, take responsibility for the integrity of the work as a whole, and have given their approval for this version to be published.

Participants. We would like to thank the patients who gave their views, though informal, on managing insulin as we prepared this manuscript. We thank the patient who consented to have a photo of the thigh with insulin tattoos.

Disclosures. Bahendeka Silver, Kaushik Ramaiya, Swai Babu Andrew, Otieno Fredrick,
Sarita Bajaj, Sanjay Kalra, Bavuma Charlotte, Karigire Claudine, and Anthony Makhoba declare that they have no competing conflict of interest.

Compliance with Ethics Guidelines. This guideline is based on previously conducted studies and does not contain any studies with human participants or animals performed by any of the authors. In drawing up the East African Diabetes Study Group (EADSG) Guidelines: Insulin Therapy in Diabetes, the authors adhered to the international and ethical standards for developing clinical practice guidelines (CPG). In compliance with the Uganda National Council of Science and Technology, the photo shown in Fig. 2 was taken with written consent of the patient embedded in his clinical notes and is anonymized.

External Reviewers. We thank the following external reviewers for their very timely and useful comments: Uloko Andrew, Shimjee Suleman, Coetzee Ankia, Ruder Sundeep, Megallaa Magdy, Khalid Shaikh, Sandeep Chaudry, Hamed Farooqi, Rayaz Malik, Dina Shrestha, Abbas Raza, Ashok Kumar Das, Noel Somasundaram, Faruque Pathan, Ko Ko, Ali Latheef, and Cecilia Jimeno.

Data Availability. The literature reviewed and analyzed during the current study is available from the corresponding author on reasonable request.

Disclaimer. Springer Healthcare is not responsible for the validity of guidelines it publishes.

Open Access. This article is distributed under the terms of the Creative Commons Attribution-NonCommercial 4.0 International License (http://creativecommons.org/licenses/ by-nc/4.0/), which permits any noncommercial use, distribution, and reproduction in any medium, provided you give appropriate credit to the original author(s) and the source, provide a link to the Creative Commons license, and indicate if changes were made. 


\section{REFERENCES}

1. International Diabetes Federation. Diabetes atlas8th edition. http://www.diabetesatlas.org/ resources/2017-atlas.html. Accessed Dec 19, 2017.

2. Institute for Health Metrics and Evaluation Human Development Network. The World Bank. The global burden of disease: generating evidence, guiding policy-Sub-Saharan Africa Regional Edition. Seattle: IHME; 2013.

3. UK Prospective Diabetes Study (UKPDS) Group. Effect of intensive blood-glucose control with metformin on complications in overweight patients with type 2 diabetes (UKPDS 34). Lancet. 1998;352(9131):854-65.

4. UK Prospective Diabetes Study (UKPDS) Group. Intensive blood-glucose control with sulphonylureas or insulin compared with conventional treatment and risk of complications in patients with type 2 diabetes (UKPDS 33). Lancet. 1998;352(9131):837-53.

5. The Diabetes Control and Complications Trial Research Group. The effect of intensive treatment of diabetes on the development and progression of long-term complications in insulin-dependent diabetes mellitus. N Engl J Med. 1993;329(14):977-86.

6. Bolen S, Tseng E, Hutfless S, et al. Diabetes medications for adults with type 2 diabetes: an update. Comparative effectiveness review no. 173. (Prepared by the Johns Hopkins University Evidence-based Practice Center under Contract No. 290-201200007-I.). AHRQ Publication No.16-EHC013-EF. Rockville: Agency for Healthcare Research and Quality; 2016. http://www.effectivehealthcare. ahrq.gov/reports/final.cfm.

7. Wirtz VJ, Knox R, Cao C, Mehrtash H, Posner NW. Insulin market profile. Netherlands: Health Action International; 2016.

8. Kalra S, Baruah MP, Sahay R, Kishor K. Pentads and hexads in diabetes care: numbers as targets; numbers as tools. Indian $\mathrm{J}$ Endocrinol Metab. 2017;21(6):794.

9. Lachin JM, Genuth S, Cleary P, Davis MD, Nathan DM. Retinopathy and nephropathy in patients with type 1 diabetes four years after a trial of intensive therapy. N Engl J Med. 2000;342(6):381-9.

10. American Diabetes Association. Standards of medical care in diabetes-2018. Diabetes Care. 2018;41(1):S1-155.

11. Damodaran GK, Gilsa E, Andrews M, Mathew C, George J, Ajith V. Apo lipoprotein B, total cholesterol, HDL-C, non HDL-C, LDL-C and triglycerides as risk factors of atherosclerotic vascular disease. Int J Adv Res. 2015;3(2):814-9.

12. Heine RJ, Balkau B, Ceriello A, Del Prato S, Horton ES, Taskinen MR. What does postprandial hyperglycaemia mean? Diabet Med. 2004;21(3):208-13.

13. Ohkubo Y, Kishikawa H, Araki E, et al. Intensive insulin therapy prevents the progression of diabetic microvascular complications in Japanese patients with non-insulin-dependent diabetes mellitus: a randomized prospective 6-year study. Diabetes Res Clin Pract. 1995;28(2):103-17.

14. ADVANCE Collaborative Group, Patel A, MacMahon $\mathrm{S}$, et al. Intensive blood glucose control and vascular outcomes in patients with type 2 diabetes. N Engl J Med. 2008;358(24):2560-72.

15. Ismail-Beigi F, Craven T, Banerji MA, et al. Effect of intensive treatment of hyperglycaemia on microvascular outcomes in type 2 diabetes: an analysis of the ACCORD randomised trial. Lancet. 2010;376(9739):419-30.

16. Saremi A, Moritz TE, Anderson RJ, et al. Rates and determinants of coronary and abdominal aortic artery calcium progression in the Veterans Affairs Diabetes Trial (VADT). Diabetes Care. 2010;33(12):2642-7.

17. Handelsman Y, Bloomgarden ZT, Grunberger G, et al. American Association of Clinical Endocrinologists and American College of Endocrinologyclinical practice guidelines for developing a diabetes mellitus comprehensive care plan-2015. Endocr Pract. 2015;21(Suppl 1):1-87.

18. Duckworth W, Abraira C, Moritz T, et al. Glucose control and vascular complications in veterans with type 2 diabetes. N Engl J Med. 2009;360(2):129-39.

19. Kahn CR, Weir GC, King GL, Jacobson AM, Moses AC, Smith RJ. Joslin: Diabetes Melito-14. Artmed Editora; 2009.

20. Turner R. Intensive blood-glucose control with sulphonylureas or insulin compared with conventional treatment and risk of complications in patients with type 2 diabetes (UKPDS 33) (vol 352, pg 837, 1998). Lancet. 1999;354(9178):602.

21. Reuven Y, Dreiher J, Shvartzman P. The prevalence of diabetes, hypertension and obesity among immigrants from East Africa and the former Soviet Union: a retrospective comparative 30-year cohort study. Cardiovasc Diabetol. 2016;15:74.

22. Bahendeka S, Wesonga R, Mutungi G, Muwonge J, Neema S, Guwatudde D. Prevalence and correlates of diabetes mellitus in Uganda: a population-based 
national survey. Trop Med Int Health. 2016;21(3):405-16.

23. Gomez-Perez FJ, Aguilar-Salinas CA, Almeda-Valdes P, Cuevas-Ramos D, Garber IL, Rull JA. HbA1c for the diagnosis of diabetes mellitus in a developing country. A position article. Arch Med Res. 2010;41:302-8.

24. Mayige M, Kagaruki G. Tanzania STEPS Survey Report. Ministry of Health and Social Welfare and National Institute for Medial Research in Collaboration with World Health Organizaation; 2013. https://www.researchgate.net/publication/ 311065616_TANZANIA_STEPS_SURVEY_REPORT. Accessed Dec 23, 2017.

25. Unnikrishnan IR, Anjana R, Mohan V. Importance of controlling diabetes early-the concept of metabolic memory, legacy effect and the case for early insulinisation. J Assoc Physicians India. 2005;53:283-7.

26. World Health Organization. World Health Organization-noncommunicable diseases (NCD) country profiles, 2014. http://www.who.int/nmh/countries/ bdi_en.pdf. Accessed Dec 23, 2017.

27. Kong M-F, Bickerton A. Insulin should be prescribed at the outset of diagnosis of type 2 diabetes. Pract Diab Int. 2011;28(2):85-7.

28. World Health Organization. Ethiopia 2015 STEPS fast sheet. http://www.who.int/chp/steps/Ethiopia 2015_STEPS_FactSheet.pdf?ua=1. Accessed on Dec 4, 2017.

29. Manne-Goehler J, Atun R, Stokes A, et al. Diabetes diagnosis and care in sub-Saharan Africa: pooled analysis of individual data from 12 countries. Lancet Diabetes Endocrinol. 2016;4(11):903-12.

30. Atun R, Davies JI, Gale EAM, et al. Diabetes in subSaharan Africa: from clinical care to health policy. Lancet Diabetes Endocrinol. 2017;5(8):622-67.

31. Katende D, Mutungi G, Baisley $\mathrm{K}$, et al. Readiness of Ugandan health services for the management of outpatients with chronic diseases. Trop Med Int Health. 2015;20(10):1385-95.

32. Vedanthan R, Kamano JH, Bloomfield GS, Manji I, Pastakia S, Kimaiyo SN. Engaging the entire care cascade in Western Kenya: a model to achieve the cardiovascular disease secondary prevention roadmap goals. Glob Heart. 2015;10(4):313-7.

33. Nnko S, Bukenya D, Kavishe BB, Biraro S, Peck R, Kapiga S, et al. Chronic diseases in North-West Tanzania and Southern Uganda. Public perceptions of terminologies, aetiologies, symptoms and preferred management. PLoS One. 2015;10(11):e0142194.

34. Nielsen J, Bahendeka SK, Bygbjerg IC, Meyrowitsch DW, Whyte SR. Accessing diabetes care in rural Uganda: economic and social resources. Glob Public Health. 2017;12(7):892-908.

35. Cook AR. Notes on the disease met with in Uganda, Central Africa. J Trop Med. 1901;4(17):5-8.

36. Amendezo E, Walker Timothy D, Karamuka V, et al. Effects of a lifestyle education program on glycemic control among patients with diabetes at Kigali University Hospital, Rwanda: a randomized controlled trial. Diabetes Res Clin Pract. 2017;126:129-37.

37. Marshall SL, Edidin D, Arena VC, et al. Prevalence and incidence of clinically recognized cases of type 1 diabetes in children and adolescents in Rwanda, Africa. Diabet Med. 2015;32(9):1186-92.

38. Ngwiri T, Were F, Predieri B, Ngugi P, Iughetti L. Glycemic control in Kenyan children and adolescents with type 1 diabetes mellitus. Int J Endocrinol. $2015 ; 2015: 761759$.

39. Majaliwa ES, Munubhi E, Ramaiya K, et al. Survey on acute and chronic complications in children and adolescents with type 1 diabetes at Muhimbili National Hospital in Dar es Salaam, Tanzania. Diabetes Care. 2007;30(9):2187-92.

40. Osujih M. Exploration of the frontiers of tradomedical practices: basis for development of alternative medical healthcare services in developing countries. J R Soc Health. 1993;113(4):190-4.

41. Agyemang C, de-Graft Aikins A, Bhopal R. Ethnicity and cardiovascular health research: pushing the boundaries by including comparison populations in the countries of origin. Ethn Health. 2012;17(6):579-96.

42. Majaliwa ES, Elusiyan BE, Adesiyun OO, et al. Type 1 diabetes mellitus in the African population: epidemiology and management challenges. Acta Biomed. 2008;79(3):255-9.

43. Muze KC, Majaliwa ES. Type 1 diabetes care updates: Tanzania. Indian J Endocrinol Metab. 2015;19(Suppl 1):S12-3.

44. McLarty D, Pollitt C, Swai A. Diabetes in Africa. Diabet Med. 1990;7(8):670-84.

45. Mwebaze RM, Kibirige D. Peripheral arterial disease among adult diabetic patients attending a large outpatient diabetic clinic at a national referral hospital in Uganda: a descriptive cross sectional study. PLoS One. 2014;9(8):e105211. 
46. Abbas ZG. Diabetic foot-an African perspective. JSM Foot Ankle. 2016;1(1):1005.

47. Byrne MM, Sturis J, Sobel RJ, Polonsky KS. Elevated plasma glucose $2 \mathrm{~h}$ postchallenge predicts defects in beta-cell function. Am J Physiol Endocrinol Metab. 1996;270(4):E572-9.

48. Owens DR, Zinman B, Bolli GB. Insulins today and beyond. Lancet. 2001;358(9283):739-46.

49. Pettus J, Santos Cavaiola T, Tamborlane WV, Edelman S. The past, present, and future of basal insulins. Diabetes Metab Res Rev. 2016;32(6):478-96.

50. Heneghan C, Badenoch D. Evidence-based medicine toolkit. New York: Wiley; 2013.

51. Garber AJ, Abrahamson MJ, Barzilay JI, et al. Consensus statement by the American Association of Clinical Endocrinologists and American College of Endocrinology on the comprehensive type 2 diabetes management algorithm-2017 executive summary. Endocr Pract. 2017;23(2):207-38.

52. International Diabetes Federation Guideline Development Group. Global guideline for type 2 diabetes. Diabetes Res Clin Pract. 2014;104(1):1-52.

53. Mohan V, Kalra S, Kesavadev J, et al. Consensus on initiation and intensification of premix insulin in type 2 diabetes management. J Assoc Physicians India. 2017;65(4):59-73.

54. Das AK, Sahay BK, Seshiah V, et al. Indian National Consensus Group: national guidelines on initiation and intensification of insulin therapy with premixed insulin analogs. Med Update. 2013;2013:227.

55. SEMDSA 2017 guidelines for the management of type 2 diabetes mellitus. J Endocrinol Metab Diabetes S Afr. 2017;22(1):S1-S196.

56. Hinson AM, Stack BC Jr. Evidence-based medicine: approach and references classification. Medical and surgical treatment of parathyroid diseases. Berlin: Springer; 2017. p. 11-3.

57. Gardner KE. 'The art of insulin treatment:' diabetes, insulin, and the 1920s. J Med Humanit. 2017:1-10.

58. Quianzon CC, Cheikh I. History of insulin. J Community Hosp Intern Med Perspect. 2012;2(2). https://doi.org/10.3402/jchimp.v2i2.18701.

59. Gill G, Yudkin JS, Tesfaye S, et al. Essential medicines and access to insulin. Lancet Diabetes Endocrinol. 2017;5(5):324-5.

60. Mayfield JA, White RD. Insulin therapy for type 2 diabetes: rescue, augmentation, and replacement of beta-cell function. Am Fam Physician. 2004;70(3):489-500.

61. Neu A, Lange K, Barrett T, et al. Classifying insulin regimens-difficulties and proposal for comprehensive new definitions. Pediatr Diabetes. 2015;16(6):402-6.

62. Guthrie DW, Guthrie RA, Hinnen D, Childs BP. It's time to abandon the sliding scale. J Fam Pract. 2011;60(5):266-70.

63. Jackson RL, Storvick WO, Hollinden CS, Stroeh LE, Stilz JG. Neutral regular insulin. Diabetes. 1972;21(4):235-45.

64. Salonen KM, Ryhänen S, Härkönen T, Ilonen J, Knip M. Autoantibodies against zinc transporter 8 are related to age, metabolic state and HLA DR genotype in children with newly diagnosed type 1 diabetes. Diabetes Metab Res Rev. 2013;29(8):646-54.

65. Home PD. The pharmacokinetics and pharmacodynamics of rapid-acting insulin analogues and their clinical consequences. Diabetes Obes Metab. 2012;14(9):780-8.

66. Kalra S. Newer basal insulin analogues: degludec, detemir, glargine. J Pak Med Assoc. 2013;63(11):1442-4.

67. Turner H, Matthews D. The use of fixed-mixture insulins in clinical practice. Eur J Clin Pharmacol. 2000;56(1):19-25.

68. Frias PF, Frias JP. New basal insulins: a clinical perspective of their use in the treatment of type 2 diabetes and novel treatment options beyond basal insulin. Curr Diabetes Rep. 2017;17(10):91.

69. NICE. Type 1 diabetes in adults: diagnosis and management. London: National Institute for Health and Care Excellence: Clinical Guidelines; 2015.

70. Canadian Diabetes Association Clinical Practice Guidelines Expert Committee, McGibbon A, Richardson C, Hernandez C, Dornan J. Pharmacotherapy in type 1 diabetes. Can J Diabetes. 2013;37(Suppl 1):S56-60.

71. Kaur KK, Allahbadia G, Singh M. An update on a etiopathogenesis and management of obesity. Obes Control Ther. 2016;3(1):1-17.

72. Nokoff N, Rewers M. Pathogenesis of type 1 diabetes: lessons from natural history studies of highrisk individuals. Ann NY Acad Sci. 2013;1281(1):1-15.

73. NICE. Diabetes (type 1 and type 2) in children and young people: diagnosis and management. London: 
National Institute for Health and Care Excellence: Clinical Guidelines; 2015.

74. Nathan DM, Cleary PA, Backlund JY, et al. Intensive diabetes treatment and cardiovascular disease in patients with type 1 diabetes. $\mathrm{N}$ Engl J Med. $2005 ; 353(25): 2643-53$.

75. Garg SK, Rosenstock J, Ways K. Optimized basalbolus insulin regimens in type 1 diabetes: insulin glulisine versus regular human insulin in combination with basal insulin glargine. Endocr Pract. 2005;11(1):11-7.

76. DeWitt DE, Hirsch IB. Outpatient insulin therapy in type 1 and type 2 diabetes mellitus: scientific review. JAMA. 2003;289(17):2254-64.

77. Chatterjee $S$, Jarvis-Kay J, Rengarajan $\mathrm{T}$, Lawrence IG, McNally PG, Davies MJ. Glargine versus NPH insulin: efficacy in comparison with insulin aspart in a basal bolus regimen in type 1 diabetes-the glargine and aspart study (GLASS) a randomised cross-over study. Diabetes Res Clin Pract. 2007;77(2):215-22.

78. De Leeuw I, Vague P, Selam JL, et al. Insulin detemir used in basal-bolus therapy in people with type 1 diabetes is associated with a lower risk of nocturnal hypoglycaemia and less weight gain over 12 months in comparison to NPH insulin. Diabetes Obes Metab. 2005;7(1):73-82.

79. Birkeland KI, Home PD, Wendisch U, et al. Insulin degludec in type 1 diabetes: a randomized controlled trial of a new-generation ultra-long-acting insulin compared with insulin glargine. Diabetes Care. 2011;34(3):661-5.

80. Chen JW, Lauritzen T, Bojesen A, Christiansen JS. Multiple mealtime administration of biphasic insulin aspart 30 versus traditional basal-bolus human insulin treatment in patients with type 1 diabetes. Diabetes Obes Metab. 2006;8(6):682-9.

81. Mortensen H, Kocova M, Teng LY, Keiding J, Bruckner I, Philotheou A. Biphasic insulin aspart vs. human insulin in adolescents with type 1 diabetes on multiple daily insulin injections. Pediatr Diabetes. $2006 ; 7(1): 4-10$.

82. Vella S, Buetow L, Royle P, Livingstone S, Colhoun HM, Petrie JR. The use of metformin in type 1 diabetes: a systematic review of efficacy. Diabetologia. 2010;53(5):809-20.

83. Holman RR, Paul SK, Bethel MA, Matthews DR, Neil HA. 10-year follow-up of intensive glucose control in type 2 diabetes. $\mathrm{N}$ Engl $\mathrm{J}$ Med. 2008;359(15):1577-89.
84. Kalra S, Gupta Y. Insulin initiation: bringing objectivity to choice. J Diabetes Metab Disord. 2015;14:17.

85. Hermansen K, Davies $M$, Derezinski $T$, Martinez Ravn G, Clauson P, Home P. A 26-week, randomized, parallel, treat-to-target trial comparing insulin detemir with NPH insulin as add-on therapy to oral glucose-lowering drugs in insulin-naive people with type 2 diabetes. Diabetes Care. 2006;29(6):1269-74.

86. Laubner K, Molz K, Kerner W, et al. Daily insulin doses and injection frequencies of neutral protamine Hagedorn (NPH) insulin, insulin detemir and insulin glargine in type 1 and type 2 diabetes: a multicenter analysis of 51964 patients from the German/Austrian DPV-wiss database. Diabetes Metab Res Rev. 2014;30(5):395-404.

87. Rosenstock J, Davies M, Home PD, Larsen J, Koenen C, Schernthaner G. A randomised, 52-week, treatto-target trial comparing insulin detemir with insulin glargine when administered as add-on to glucose-lowering drugs in insulin-naive people with type 2 diabetes. Diabetologia. 2008;51(3):408-16.

88. Unnikrishnan AG, Tibaldi J, Hadley-Brown M, et al. Practical guidance on intensification of insulin therapy with BIAsp 30: a consensus statement. Int J Clin Pract. 2009;63(11):1571-7.

89. El Naggar NK, Soewondo P, Khamseh ME, Chen JW, Haddad J. Switching from biphasic human insulin 30 to biphasic insulin aspart 30 in type 2 diabetes is associated with improved glycaemic control and a positive safety profile: results from the $A(1)$ chieve study. Diabetes Res Clin Pract. 2012;98(3):408-13.

90. Shah S, Yang W, Hasan MI, Malek R, Molskov Bech $\mathrm{O}$, Home P. Biphasic insulin aspart 30 in insulinnaive people with type 2 diabetes in non-western nations: results from a regional comparative multinational observational study (A(1)chieve). Diabetes Technol Ther. 2013;15(11):954-63.

91. Kramer CK, Zinman B, Retnakaran R. Short-term intensive insulin therapy in type 2 diabetes mellitus: a systematic review and meta-analysis. Lancet Diabetes Endocrinol. 2013;1(1):28-34.

92. Kann PH, Wascher T, Zackova V, et al. Starting insulin therapy in type 2 diabetes: twice-daily biphasic insulin aspart 30 plus metformin versus once-daily insulin glargine plus glimepiride. Exp Clin Endocrinol Diabetes. 2006;114(9):527-32.

93. Raskin PR, Hollander PA, Lewin A, et al. Basal insulin or premix analogue therapy in type 2 diabetes patients. Eur J Intern Med. 2007;18(1):56-62.

94. Oyer DS, Shepherd MD, Coulter FC, et al. Efficacy and tolerability of self-titrated biphasic insulin 
aspart 70/30 in patients aged $>65$ years with type 2 diabetes: an exploratory post hoc subanalysis of the INITIATEplus trial. Clin Ther. 2011;33(7):874-83.

95. Strojek K, Bebakar WM, Khutsoane DT, et al. Oncedaily initiation with biphasic insulin aspart 30 versus insulin glargine in patients with type 2 diabetes inadequately controlled with oral drugs: an openlabel, multinational RCT. Curr Med Res Opin. 2009;25(12):2887-94.

96. Kalra S, Plata-Que T, Kumar D, et al. Initiation with once-daily BIAsp 30 results in superior outcome compared to insulin glargine in Asians with type 2 diabetes inadequately controlled by oral anti-diabetic drugs. Diabetes Res Clin Pract. 2010;88(3):282-8.

97. Raskin P, Allen E, Hollander P, et al. Initiating insulin therapy in type 2 diabetes: a comparison of biphasic and basal insulin analogs. Diabetes Care. 2005;28(2):260-5.

98. Buse JB, Wolffenbuttel BH, Herman WH, et al. DURAbility of basal versus lispro mix 75/25 insulin efficacy (DURABLE) trial 24-week results: safety and efficacy of insulin lispro mix 75/25 versus insulin glargine added to oral antihyperglycemic drugs in patients with type 2 diabetes. Diabetes Care. 2009;32(6):1007-13.

99. Buse JB, Wolffenbuttel BH, Herman WH, et al. The DURAbility of Basal versus Lispro mix 75/25 insulin Efficacy (DURABLE) trial: comparing the durability of lispro mix 75/25 and glargine. Diabetes Care. 2011;34(2):249-55.

100. Jothydev K, Rajput R, John M, Annamalai AK, Rao PV. Consensus statement on choice of insulin therapy in type 2 diabetes. J Assoc Physicians India. 2016;64:13-18.

101. Christiansen JS, Niskanen L, Rasmussen S, Johansen T, Fulcher G. Lower rates of hypoglycemia during maintenance treatment with insulin degludec/insulin aspart versus biphasic insulin aspart 30: a combined analysis of two phase 3 a studies in type 2 diabetes. J Diabetes. 2016;8(5):720-8.

102. Kaneko S, Chow F, Choi DS, et al. Insulin degludec/ insulin aspart versus biphasic insulin aspart 30 in Asian patients with type 2 diabetes inadequately controlled on basal or pre-/self-mixed insulin: a 26-week, randomised, treat-to-target trial. Diabetes Res Clin Pract. 2015;107(1):139-47.

103. Shi LX, Li PF, Hou JN. Differential treatment response to insulin intensification therapy: a post hoc analysis of a randomized trial comparing premixed and basal-bolus insulin regimens. Diabetes Ther. 2017;8(4):915-28.
104. Inzucchi SE, Bergenstal RM, Buse JB, et al. Management of hyperglycemia in type 2 diabetes, 2015: a patient-centered approach: update to a position statement of the American Diabetes Association and the European Association for the Study of Diabetes. Diabetes Care. 2015;38(1):140-9.

105. Mwanri AW, Kinabo J, Ramaiya K, Feskens EJ. Prevalence of gestational diabetes mellitus in urban and rural Tanzania. Diabetes Res Clin Pract. 2014;103(1):71-8.

106. Nakabuye B, Bahendeka S, Byaruhanga R. Prevalence of hyperglycaemia first detected during pregnancy and subsequent obstetric outcomes at St. Francis Hospital Nsambya. BMC Res Notes. 2017;10(1):174.

107. Muganyizi PS, Kidanto HL. Impact of change in maternal age composition on the incidence of caesarean section and low birth weight: analysis of delivery records at a tertiary hospital in Tanzania, 1999-2005. BMC Pregnancy Childbirth. 2009;9:30.

108. World Health Organization. Diagnostic criteria and classification of hyperglycaemia first detected in pregnancy. Geneva: WHO; 2013.

109. Balsells M, Garcia-Patterson A, Sola I, Roque M, Gich I, Corcoy R. Glibenclamide, metformin, and insulin for the treatment of gestational diabetes: a systematic review and meta-analysis. BMJ. 2015;350:h102.

110. Balaji V, Balaji MS, Alexander C, et al. Premixed insulin aspart 30 (BIAsp 30) versus premixed human insulin 30 (BHI 30) in gestational diabetes mellitus: a randomized open-label controlled study. Gynecol Endocrinol. 2012;28(7):529-32.

111. Stanifer JW, Jing B, Tolan S, et al. The epidemiology of chronic kidney disease in sub-Saharan Africa: a systematic review and meta-analysis. Lancet Glob Health. 2014;2(3):e174-81.

112. Iglesias P, Diez JJ. Insulin therapy in renal disease. Diabetes Obes Metab. 2008;10(10):811-23.

113. Kengne AP, Amoah AG, Mbanya JC. Cardiovascular complications of diabetes mellitus in sub-Saharan Africa. Circulation. 2005;112(23):3592-601.

114. Onyekwere CA, Ogbera AO, Balogun BO. Non-alcoholic fatty liver disease and the metabolic syndrome in an urban hospital serving an African community. Ann Hepatol. 2011;10(2):119-24.

115. Edghill EL, Flanagan SE, Patch AM, et al. Insulin mutation screening in 1,044 patients with diabetes: mutations in the INS gene are a common cause of neonatal diabetes but a rare cause of diabetes 
diagnosed in childhood or adulthood. Diabetes. 2008;57(4):1034-42.

116. Nyangabyaki-Twesigye C, Muhame MR, Bahendeka S. Permanent neonatal diabetes mellitus-a case report of a rare cause of diabetes mellitus in East Africa. Afr Health Sci. 2015;15(4):1339-41.

117. Rubio-Cabezas O, Hattersley AT, Njolstad PR, et al. ISPAD clinical practice consensus guidelines 2014. The diagnosis and management of monogenic diabetes in children and adolescents. Pediatr Diabetes. 2014;15(Suppl 20):47-64.

118. von Muhlendahl KE, Herkenhoff H. Long-term course of neonatal diabetes. $\mathrm{N}$ Engl J Med. 1995;333(11):704-8.

119. Salti I, Benard E, Detournay B, et al. A populationbased study of diabetes and its characteristics during the fasting month of Ramadan in 13 countries: results of the epidemiology of diabetes and Ramadan 1422/2001 (EPIDIAR) study. Diabetes Care. 2004;27(10):2306-11.

120. Jabbar A, Hassanein M, Beshyah SA, Boye KS, Yu M, Babineaux SM. CREED study: hypoglycaemia during Ramadan in individuals with type 2 diabetes mellitus from three continents. Diabetes Res Clin Pract. 2017;132:19-26.

121. Al-Arouj M, Assaad-Khalil S, Buse J, et al. Recommendations for management of diabetes during Ramadan: update 2010. Diabetes Care. 2010;33(8):1895-902.

122. Hassanein M, Al-Arouj M, Hamdy O, et al. Diabetes and Ramadan: practical guidelines. Diabetes Res Clin Pract. 2017;126:303-16.

123. Grunberger G. Insulin analogs-are they worth it? Yes! Diabetes Care. 2014;37(6):1767-70.

124. Akram J, De Verga V. Insulin lispro (Lys(B28), Pro(B29) in the treatment of diabetes during the fasting month of Ramadan. Ramadan Study Group. Diabet Med. 1999;16(10):861-6.

125. Mattoo V, Milicevic Z, Malone JK, et al. A comparison of insulin lispro Mix25 and human insulin $30 / 70$ in the treatment of type 2 diabetes during Ramadan. Diabetes Res Clin Pract. 2003;59(2):137-43.

126. Hui E, Bravis V, Salih S, Hassanein M, Devendra D. Comparison of Humalog Mix 50 with human insulin Mix 30 in type 2 diabetes patients during Ramadan. Int J Clin Pract. 2010;64(8):1095-9.

127. Bakiner O, Ertorer ME, Bozkirli E, Tutuncu NB, Demirag NG. Repaglinide plus single-dose insulin glargine: a safe regimen for low-risk type 2 diabetic patients who insist on fasting in Ramadan. Acta Diabetol. 2009;46(1):63-5.

128. Shehadeh N, Maor Y, Ramadan Study Group. Effect of a new insulin treatment regimen on glycaemic control and quality of life of Muslim patients with type 2 diabetes mellitus during Ramadan fast-an open label, controlled, multicentre, cluster randomised study. Int J Clin Pract. 2015;69(11):1281-8.

129. Soeatmadji DW, Sanusi H, Adam JMF, Soewondo P. A multicenter, prospective, non-interventional evaluation of efficacy and safety of using biphasic insulin aspart as monotherapy, or in combination with oral hypoglycemic agent, in the treatment of type 2 diabetic patients before, during, and after Ramadan. J Indones Med Assoc. 2009;59(12).

130. Kassem HS, Zantout MS, Azar ST. Insulin therapy during Ramadan fast for type 1 diabetes patients. J Endocrinol Invest. 2005;28(9):802-5.

131. Benbarka MM, Khalil AB, Beshyah SA, Marjei S, Awad SA. Insulin pump therapy in Moslem patients with type 1 diabetes during Ramadan fasting: an observational report. Diabetes Technol Ther. 2010;12(4):287-90.

132. Acquah S, Boampong JN, Eghan Jnr BA, Eriksson M. Evidence of insulin resistance in adult uncomplicated malaria: result of a two-year prospective study. Malar Res Treat. 2014;2014:136148.

133. Mohapatra MK. Profile of severe falciparum malaria in diabetics. Int J Diabetes Dev Ctries. 2001;21:156-61.

134. Kalra S, Aggarwal S, Khandelwal D, Dutta D. Management of glycemia in acute febrile illness. Indian J Endocrinol Metab. 2017;21(3):460-3.

135. Jacobi J, Bircher N, Krinsley J, et al. Guidelines for the use of an insulin infusion for the management of hyperglycemia in critically ill patients. Crit Care Med. 2012;40(12):3251-76.

136. Larson R, Capili B, Eckert-Norton M, Colagreco JP, Anastasi JK. Disorders of glucose metabolism in the context of human immunodeficiency virus infection. J Am Acad Nurse Pract. 2006;18(3):92-103.

137. Kalra S, Kalra B, Agrawal N, Unnikrishnan A. Understanding diabetes in patients with HIV/AIDS. Diabetol Metab Syndr. 2011;3(1):2.

138. Sobngwi E, Vexiau P, Levy V, et al. Metabolic and immunogenetic prediction of long-term insulin remission in African patients with atypical diabetes. Diabet Med. 2002;19(10):832-5. 
139. Umpierrez GE, Smiley D, Kitabchi AE. Narrative review: ketosis-prone type 2 diabetes mellitus. Ann Intern Med. 2006;144(5):350-7.

140. Murata GH, Shah JH, Hoffman RM, et al. Intensified blood glucose monitoring improves glycemic control in stable, insulin-treated veterans with type 2 diabetes: the Diabetes Outcomes in Veterans Study (DOVES). Diabetes Care. 2003;26(6):1759-63.

141. DeVries JH. Postprandial or fasting hyperglycemia: time to move on? Diabetes Technol Ther. 2015;17(7):441-2.

142. Wambui Charity K, Kumar AMV, Hinderaker SG, Chinnakali P, Pastakia SD, Kamano J. Do diabetes mellitus patients adhere to self-monitoring of blood glucose (SMBG) and is this associated with glycemic control? Experiences from a SMBG program in western Kenya. Diabetes Res Clin Pract. 2016;112:37-43.

143. Institute For Safe Medication Practices. ISMP guidelines for optimizing safe subcutaneous insulin use in adults. 2017. https://www.ismp.org/tools/ guidelines/Insulin-Guideline.pdf. Accessed 4 Dec 2018.

144. Wako A, Belay S, Feleke Y, Kebede T. Assessment of the magnitude, severity and associated factors of hypoglycemia in diabetic patients attending National Diabetes Referral Clinic at Tikur Anbessa Hospital, Addis Ababa, Ethiopia. J Diabetes Metab. 2017;8:471.

145. Liu SC, Tu YK, Chien MN, Chien KL. Effect of antidiabetic agents added to metformin on glycaemic control, hypoglycaemia and weight change in patients with type 2 diabetes: a network metaanalysis. Diabetes Obes Metab. 2012;14(9):810-20.

146. Almaatouq MA, Al-Arouj M, Amod A, et al. Barriers to the delivery of optimal antidiabetic therapy in the Middle East and Africa. Int J Clin Pract. 2014;68(4):503-11.

147. Otieno CF, Kanu JE, Karari EM, Okech-Helu V, Joshi MD, Mutai K. Glucose-lowering therapies, adequacy of metabolic control, and their relationship with comorbid depression in outpatients with type 2 diabetes in a tertiary hospital in Kenya. Diabetes Metab Syndr Obes. 2017;10:141-9.

148. Moghissi ES, Korytkowski MT, DiNardo M, et al. American Association of Clinical Endocrinologists and American Diabetes Association consensus statement on inpatient glycemic control. Diabetes Care. 2009;32(6):1119-31.

149. International Hypoglycaemia Study Group. Glucose concentrations of less than $3.0 \mathrm{mmol} / \mathrm{L}(54 \mathrm{mg} / \mathrm{dL})$ should be reported in clinical trials: a joint position statement of the American Diabetes Association and the European Association for the Study of Diabetes. Diabetes Care. 2017;40(1):155-7.

150. Giugliano D, Chiodini P, Maiorino MI, Bellastella G, Esposito K. Intensification of insulin therapy with basal-bolus or premixed insulin regimens in type 2 diabetes: a systematic review and meta-analysis of randomized controlled trials. Endocrine. 2016;51(3):417-28.

151. Bellido V, Suarez L, Rodriguez MG, et al. Comparison of basal-bolus and premixed insulin regimens in hospitalized patients with type 2 diabetes. Diabetes Care. 2015;38(12):2211-6.

152. Rehman HU, Mohammed K. Perioperative management of diabetic patients. Curr Surg. 2003;60(6):607-11.

153. Meneghini LF. Perioperative management of diabetes: translating evidence into practice. Cleve Clin J Med. 2009;76(Suppl 4):S53-9.

154. Corsino L, Dhatariya K, Umpierrez G. Management of diabetes and hyperglycemia in hospitalized patients. In: De Groot LJ, Chrousos G, Dungan K, Feingold KR, Grossman A, Hershman JM, et al., editors. Endotext: South Dartmouth; 2000.

155. Brady VJ. Insulin therapy: the old, the new and the novel-an overview. Nurs Clin North Am. 2017;52(4):539-52.

156. Ogle GD, Abdullah M, Mason D, Januszewski AS, Besancon S. Insulin storage in hot climates without refrigeration: temperature reduction efficacy of clay pots and other techniques. Diabet Med. 2016;33(11):1544-53.

157. Frid AH, Kreugel G, Grassi G, et al. New insulin delivery recommendations. Mayo Clin Proc. 2016;91(9):1231-55.

158. Elisabetta R, Prüss-Ustün HY. Assessing the burden of disease from sharps injuries to healthcare workers at national and local levels. Geneva: World Health Organization; 2005. p. 11.

159. Heinemann L. Biosimilar insulins. Expert Opin Biol Ther. 2012;12(8):1009-16.

160. Bommer C, Heesemann E, Sagalova V, et al. The global economic burden of diabetes in adults aged 20-79 years: a cost-of-illness study. Lancet Diabetes Endocrinol. 2017;5(6):423-30.

161. Kamara NT, Asiimwe S. Dyslipidaemia and hypertension among adults with diabetes in rural Uganda. Trop Doct. 2010;40(1):41-2. 
162. Cheung BM, Ong KL, Cherny SS, Sham P-C, Tso AW, Lam KS. Diabetes prevalence and therapeutic target achievement in the United States, 1999 to 2006. Am J Med. 2009;122(5):443-53.

163. Brown JB, Nichols GA, Perry A. The burden of treatment failure in type 2 diabetes. Diabetes Care. 2004;27(7):1535-40.

164. ORIGIN Trial Investigators. Basal insulin and cardiovascular and other outcomes in dysglycemia. N Engl J Med. 2012;2012(367):319-28.

165. Li Y, Xu W, Liao Z, et al. Induction of long-term glycemic control in newly diagnosed type 2 diabetic patients is associated with improvement of $\beta$-cell function. Diabetes Care. 2004;27(11):2597-602.

166. Weng J, Li Y, Xu W, et al. Effect of intensive insulin therapy on $\beta$-cell function and glycaemic control in patients with newly diagnosed type 2 diabetes: a multicentre randomised parallel-group trial. Lancet. 2008;371(9626):1753-60.

167. Hu Y, Li L, Xu Y, et al. Short-term intensive therapy in newly diagnosed type 2 diabetes partially restores both insulin sensitivity and $\beta$-cell function in subjects with long-term remission. Diabetes Care. 2011;34(8):1848-53. 